\title{
Time-Dependent Fully Nonlinear Geostrophic Adjustment
}

\author{
Allen C. Kuo and Lorenzo M. Polvani \\ Program in Applied Mathematics and Department of Applied Physics, Columbia University, New York, New York
}

(Manuscript received 12 August 1996, in final form 19 December 1996)

\begin{abstract}
Shock-capturing numerical methods are employed to integrate the fully nonlinear, rotating 1D shallow-water equations starting from steplike nongeostrophic initial conditions (a Rossby adjustment problem). Such numerical methods allow one to observe the formation of multiple bores during the transient adjustment process as well as their decay due to rotation. It is demonstrated that increasing the rotation and/or the nonlinearity increases the rate of decay. Additionally, the time required for adjustment to be completed and its dependence on nonlinearity is examined; this time is found to be highly measure dependent. Lastly, the final adjusted state of the system is observed through long time integrations. Although the bores that form provide a mechanism for dissipation, their decay results in a final state in very good agreement with the one computed by well-known (dissipationless) conservation methods.
\end{abstract}

\section{Introduction}

Geostrophic adjustment in rotating fluids is the mutual adaptation of mass and momentum toward a steady geostrophic state. In this paper we employ numerical techniques developed for gas dynamics to study the fully nonlinear time-dependent geostrophic adjustment problem. We carefully investigate both the early and the late stages of adjustment. To motivate the present work, we start with a brief summary of the subject (see Blumen 1972).

The adjustment problem was first considered by Rossby (1938). Using conservation of mass and momentum, he derived the geostrophically balanced steady end state for an ocean to which momentum is impulsively imparted. Since then, many studies have employed variations of Rossby's technique (conservation of mass, linear and angular momentum, potential vorticity $\mathrm{PV}$, etc.) to determine time-independent end states in a variety of contexts (Mihaljan 1963; Csanady 1971,1979; Gill 1976; Flierl 1979; Middleton 1987; Schubert et al. 1980; Stommel and Veronis 1980; Hsueh and Cushman-Roisin 1983; Ou 1983,1984; van Heijst 1985; McWilliams 1988; Glendening 1993; Boss and Thompson 1995).

Common to these papers is that the systems studied possess less energy in their end states than they do initially - a fact originally noted by Rossby. This question was indirectly addressed by Cahn (1945),

Corresponding author address: Dr. Lorenzo M. Polvani, Department of Applied Physics, Columbia University, Seeley W. Mudd Bldg., Room 209, 500 West 120th Street, New York, NY 10027.

E-mail: polvani@columbia.edu who solved the linear time-dependent initial value problem and showed how the end state is achieved through decaying inertial oscillations, which disperse energy away in pulses of Poincaré waves. Further initial value problems with related linearized systems have confirmed this scenario (Obukhov 1949; Bolin 1953; Washington 1964; Gill 1976; Hermann and Owens 1993).

However, the fully nonlinear time-dependent picture of the adjustment problem is as yet incomplete. Blumen (1967b) considered the effects of first-order nonlinear corrections and showed how they lead to a spatial shift in the solution and a delay in the adjustment process. Killworth (1992) examined the collapse of a fluid column using fully nonlinear equations; he showed how nonlinearity gives rise to wave breaking, which in turn acts as a mechanism for energy, and thus PV, dissipation. Killworth's study therefore prompts the question, If energy dissipation is present, can one use conservation methods to determine the adjusted end states?

To address this question, we revisit the adjustment problem first studied by Gill (1976), who considered the evolution of an initial step in the height field of a one-layer, rotating, shallow-water fluid originally at rest. The reason for choosing this simplest (if not physically most realistic) adjustment problem is that it can be directly related to its nonrotating counterpart, the so-called dam break problem (Stoker 1958), which in turn is the incompressible version of the classic "Riemann problem" (1858) of gas dynamics. The connection between the shallow-water equations and gas dynamics (Riabouchinsky 1932) allows us to make use of sophisticated numerical techniques developed for capturing shocks (in our case, bores) and to directly iden- 
tify the effects of rotation and nonlinearity on their formation and evolution.

By extending the Gill problem to the nonlinear regime, we establish in this paper that 1) bores can form even in the presence of rotation (see also Houghton 1969) and, more interestingly, that 2) they decay due to the same rotation and thus 3 ) produce only a small dissipative effect on the overall adjustment process, hence 4) validating the use of conservation laws to determine the balanced end states.

These results are presented as follows. We start in section 2 by defining the governing equations, discussing the appropriate scalings, and outlining the numerical procedure. In section 3 , we introduce the qualitative effects of nonlinearity and rotation on the adjustment process, and compare rotating nonlinear adjustment with its nonrotating and linear counterparts. In section 4, we quantify the dependence of the solution on rotation and nonlinearity. In section 5, we perform an asymptotic analysis on the adjustment problem in the weak rotation limit in order to shed some light on the mechanism of bore decay. In section 6 , we present the results of longtime integrations. In particular, we discuss the question of how long adjustment takes to complete, examine the dependence of the adjustment time on nonlinearity, and compare our computed long-time nearly steady solutions to the adjusted end states predicted by Boss and Thompson (1995). Finally, in section 7 we show how bores may emerge in a rotating environment from smooth initial conditions.

\section{Basic equations, scaling, and numerical procedure}

\section{a. Governing equations}

The governing equations for a single, inviscid, homogeneous shallow layer of fluid rotating with constant angular velocity follow from the conservation of mass and momentum. The three dependent variables describing the motion of such a fluid are $u$ and $v$, the velocities in the $x$ and $y$ directions, respectively, and $h$, the height of the fluid layer. In dimensional form the governing shallow-water equations are

$$
\begin{array}{r}
\frac{\partial u}{\partial t}+u \frac{\partial u}{\partial x}+g \frac{\partial h}{\partial x}-f v=0 \\
\frac{\partial v}{\partial t}+u \frac{\partial v}{\partial x}+f u=0 \\
\frac{\partial h}{\partial t}+\frac{\partial(h u)}{\partial x}=0
\end{array}
$$

where $g$ is the gravitational acceleration and $f$ is twice the angular velocity of rotation. In this paper, we deal only with initial conditions that are independent of $y$; if $(u, v, h)$ are independent of $y$ at $t=0$, they remain so for all $t>0$, justifying the neglect of $y$ derivatives in the governing equations.

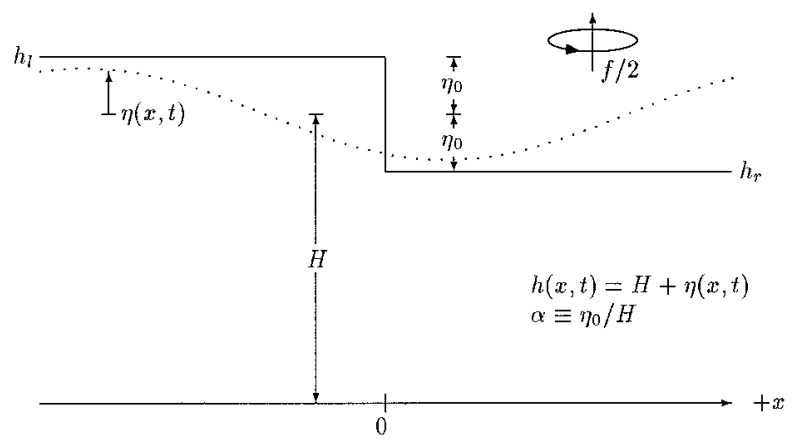

FIG. 1. Schematic of the height field. The upper solid line denotes the fluid surface at $t=0$.

We study the time evolution of an initially motionless fluid layer with a discontinuity in the height field, so that at $t=0$

$$
\begin{aligned}
& u(x, t=0)=0 \\
& v(x, t=0)=0 \\
& h(x, t=0)= \begin{cases}h_{l} \equiv H+\eta_{0}, & x<0 \\
h_{r} \equiv H-\eta_{0}, & x>0,\end{cases}
\end{aligned}
$$

where $h_{l}$ and $h_{r}$ are the heights to the left and right of the discontinuity. The initial condition is illustrated in Fig. 1. Instead of $h$, it is convenient to use as a third independent variable the perturbation height $\eta \equiv h-H$.

\section{b. Scaling}

We nondimensionalize the governing equations as follows:

$$
\begin{aligned}
\eta & =\eta_{0} \eta^{\prime} \\
u & =\left(\eta_{0} / H\right) \sqrt{g H} u^{\prime} \\
v & =\left(\eta_{0} / H\right) \sqrt{g H} v^{\prime} \\
x & =L x^{\prime} \\
t & =(L / \sqrt{g H}) t^{\prime} .
\end{aligned}
$$

The scale for $u$ and $v$ is chosen so that the appropriate linear equations are recovered in the limit $\eta_{0} \ll H$. The choice of scale for $t$ is dictated by the fact that we want to capture both the rotating and nonrotating cases with a single scaling [the obvious choice $t=$ $(1 / f) t^{\prime}$ fails when $\left.f=0\right]$. With these scalings, the nondimensional governing equations become, dropping primes,

$$
\begin{array}{r}
\frac{\partial u}{\partial t}+\alpha\left(u \frac{\partial u}{\partial x}\right)+\frac{\partial \eta}{\partial x}-\epsilon v=0 \\
\frac{\partial v}{\partial t}+\alpha\left(u \frac{\partial v}{\partial x}\right)+\epsilon u=0 \\
\frac{\partial \eta}{\partial t}+\frac{\partial u}{\partial x}+\alpha \frac{\partial \eta u}{\partial x}=0
\end{array}
$$


where $\alpha \equiv \eta_{0} / H$ and $\epsilon \equiv f L / \sqrt{g H}$. The nondimensional initial conditions become

$$
\begin{aligned}
& u(x, t=0)=0 \\
& v(x, t=0)=0 \\
& \eta(x, t=0)= \begin{cases}1, & x<0 \\
-1, & x>0 .\end{cases}
\end{aligned}
$$

Two parameters appear in the nondimensional equations: $\alpha$ controls the nonlinearity, and $\epsilon$ controls the rotation. It is worth noting that, since our initial condition does not possess an intrinsic length scale, both Eqs. (1) and (2) - and thus their solutions-remain invariant under the transformation $[f \rightarrow a f, t \rightarrow$ $t / a, x \rightarrow x / a$ ], for any constant $a$. This implies that the quantitative effects of rotation can be incorporated into the choice of time and length scales. Hence, except for the case $f=0$, one can set, without loss of generality, $\epsilon=1$; this is equivalent to choosing (as expected) $L=\sqrt{g H} / f \equiv L_{d}$ (the Rossby deformation radius) and scaling $t$ with $(1 / f)$. In the special case $f$ $=0$, there is no intrinsic length scale; our scaling (3) remains valid, and $\epsilon=0$.

\section{c. Numerical procedure}

Since the shallow-water equations admit discontinuous solutions (shock/bores) and such solutions are a main focus of this paper, a numerical method specifically designed to capture discontinuities is necessary (simple schemes tend to be either too dissipative or too oscillatory in the vicinity of discontinuities). We have used a Roe approximate Riemann solver with second-order flux-limiting corrections. This finite difference scheme breaks up the global problem into a set of local Riemann problems, which are then approximately solved with a method due to Roe (1981). We have used the CLAWPACK implementation of this scheme (Conservation LAWs software PACKage, version 2.0; LeVeque 1995). CLAWPACK provides routines to solve systems of hyperbolic equations, usually written in conservation law form. The use of conservation law form provides a guarantee that shock/bore speeds are captured correctly ${ }^{1}$; nonconservative methods have no such guarantee (Lax and Wendroff 1960; Leveque 1992). The Coriolis terms do not affect this result. The shallow-water equations (1) cast in this form are (Cahn 1945)

\footnotetext{
${ }^{1}$ In using the word "correctly," we have assumed that all energy losses due to bores occur in the same layer in which they are formed. This assumption is appropriate in a single-layer problem, but for the problem of an internal (lower layer) bore in a two-layer Boussinesq system, conservation law form may not be appropriate since energy loss can take place in the less dense upper layer (see Klemp et al. 1996).
}

$$
\begin{aligned}
\frac{\partial(h u)}{\partial t}+\frac{\partial\left(h u^{2}+(1 / 2) g h^{2}\right)}{\partial x}-f h v & =0 \\
\frac{\partial(h v)}{\partial t}+\frac{\partial(h u v)}{\partial x}+f h u & =0 \\
\frac{\partial h}{\partial t}+\frac{\partial(h u)}{\partial x} & =0 .
\end{aligned}
$$

In appendix A we describe the numerical method in more detail and provide some test results on the accuracy of CLAWPACK.

\section{General features of nonlinear geostrophic adjustment}

Before presenting the key features that characterize nonlinear geostrophic adjustment, we briefly review three simpler initial value problems in an effort to isolate the effects of rotation $(\epsilon \neq 0)$ and nonlinearity $(\alpha \neq 0)$ on the evolution of the flow. Hence we consider first (i) the linear nonrotating problem $(\alpha=\epsilon$ $=0)$, (ii) the nonlinear nonrotating problem $(\alpha \neq 0$, $\epsilon=0)$, and (iii) the linear rotating problem ( $\alpha=0$, $\epsilon \neq 0$.). In each case we recall the exact solution, and we note the qualitative features of the evolution, the amplitude and speed of the discontinuities, and the final state of the system.

\section{a. The linear nonrotating initial value problem}

With $\alpha=\epsilon=0$, Eqs. (4) with initial conditions (5) admit the well-known D'Alembert solution

$$
\begin{aligned}
& u(x, t)= \begin{cases}1 & \text { for }|x|<t \\
0 & \text { for }|x|>t\end{cases} \\
& v(x, t)=0 \forall(x, t) \quad t>0
\end{aligned}
$$

This solution is a function of the similarity variable $x / t$. We plot $\eta$ in Fig. 2a at a few select times. Two discontinuities, whose height (dimensionally $\eta_{0}$ ) is constant in time, propagate outward at the nondimensional speed of 1 (dimensionally $\sqrt{g H}$ ). It is important to note that these discontinuities are not bores, in the sense that the characteristic lines (see Stoker 1958) of the underlying equations (in the same family) do not intersect in the $x-t$ plane. At long times both the velocity and height fields are constant. 


\section{b. The nonlinear nonrotating initial value problem}

With $\epsilon=0$ but $\alpha \neq 0$, Eqs. (4) with initial conditions (5) reduce to the dam break problem, for which the exact analytic solution ${ }^{2}$ is (Stoker 1958)

$$
\begin{aligned}
& u(x, t)= \begin{cases}0 & \text { region } \mathrm{I} \\
\frac{2}{3 \alpha}\left(c_{l}+x / t\right) & \text { region } \mathrm{II} \\
\bar{u} & \text { region } \mathrm{III} \\
0 & \text { region } \mathrm{IV}\end{cases} \\
& v(x, t)=0 \forall(x, t) \quad t>0
\end{aligned}
$$

where the values of $\bar{u}, \bar{\eta}$, and the bore speed $c_{s}$ are obtained by solving

$$
\begin{aligned}
& \bar{u}=\frac{1}{\alpha}\left[c_{s}-\frac{c_{r}^{2}}{4 c_{s}}-c_{r}\left(\frac{c_{r}^{2}}{16 c_{s}}+\frac{1}{2}\right)^{1 / 2}\right] \\
& \bar{\eta}=\frac{1}{\alpha}\left[\frac{c_{s} c_{r}^{2}}{\left(c_{s}-\alpha \bar{u}\right)}-1\right] \\
& 1=\frac{\alpha \bar{u}+2 \bar{c}}{2 c_{l}} .
\end{aligned}
$$

Here $\bar{c} \equiv(1+\alpha \bar{\eta})^{1 / 2}$ and the constants $c_{l} \equiv(1+\alpha)^{1 / 2}$ and $c_{r} \equiv(1-\alpha)^{1 / 2}$ are, respectively, the nondimensional linear gravity wave speeds to the left and to the right of the initial discontinuity. The four regions of the solution are illustrated in Fig. 3 and are given by region I: $x<c_{l} t$, region II: $c_{l} t<x<(\alpha \bar{u}-\bar{c}) t$, region III: $(\alpha \bar{u}-\bar{c}) t<x c_{s} t$, region IV: $x>c_{s} t$. The bore demarcates the boundary between regions III and IV. Region II contains a "rarefaction" wave.

This solution, albeit nonlinear, is also a function of the similarity variable $x / t$. For a case with $\alpha=0.3$, we plot $\eta$ in Fig. $2 b$ at a few select times. The left-right symmetry of the solution is broken by the nonlinearity (cf. Fig. 2a). In this case, only a single discontinuity propagates outward to the right at constant height $\bar{\eta}$ and speed $c_{s}$; here this discontinuity is truly a bore, in the sense that one set of characteristics does intersect. The other set does not cross, leading to the formation of the rarefaction wave propagating to the left. At long times, both $u$ and $\eta$ are constant, and their values, respectively $\bar{u}$ and $\bar{\eta}$, are set by the degree of nonlinearity $\alpha$.

\footnotetext{
${ }^{2}$ We note that Eq. (10.8.3) on p. 336 of Stoker (1958) is probably incorrect, since it does not follow from Stoker's own procedure and is irreconcilable with his Fig. 10.8.3 in the limit $c_{s} \rightarrow c_{r}$.
}

\section{c. Linear rotating initial value problem}

With $\epsilon=1$ but $\alpha=0$, Eqs. (4) with initial conditions (5) give the linear geostrophic adjustment problem first studied by Gill (1976). The solution in that case is

$$
\begin{aligned}
& u(x, t)= \begin{cases}J_{0}\left(\sqrt{t^{2}-x^{2}}\right) & \text { for }|x|<t \\
0 & \text { for }|x|>t\end{cases} \\
& v(x, t)= \begin{cases}\frac{2}{\pi} \int_{0}^{\infty} \frac{\cos k x \cos \left(\sqrt{1+k^{2}}\right) t}{k^{2}+1} d k & \text { for }|x|<t \\
0 & \text { for }|x|>t\end{cases} \\
& \eta(x, t)=\left\{\begin{array}{cc}
-\frac{2}{\pi} \int_{0}^{\infty} \frac{k \sin k x \cos \left(\sqrt{1+k^{2}}\right) t}{k^{2}+1} d k \\
-1 & \text { for }|x|<t
\end{array}\right.
\end{aligned}
$$

where $J_{0}$ is the Bessel function of order zero.

Because of rotation, the solution is now a function of the new similarity variable $\sqrt{t^{2}-x^{2}}$. We plot $\eta$ in Fig. 2c at a few select times. First, the solution is symmetric because the problem is linear. Second, the two identical discontinuities propagating outward at constant speed 1 (dimensionally $\sqrt{g H}$ ) and constant height 1 (dimensionally $\eta_{0}$ ) are not bores (characteristics do not cross). At long times, the key effect of rotation is apparent by contrasting Fig. 2c with Fig. 2a: once the Poincaré waves have left the domain, the height of the fluid surface is not flat but assumes the characteristic slant of a geostrophically balanced flow (Gill 1976).

\section{d. Nonlinear rotating initial value problem}

With $\epsilon=1$ and $\alpha \neq 0$, Eqs. (4) with initial conditions (5) give the fully nonlinear geostrophic adjustment problem. Analytic solutions in this case are difficult to find; a nonlinear analysis along the lines of Stoker's (1958) fails due to the complicated bending and intersection of characteristics in the $x-t$ plane. Therefore, we have resorted to numerical techniques to obtain solutions (cf. section 2c and appendix A).

An example of fully nonlinear rotating adjustment is presented in Fig. 2d, where we have plotted $\eta$ at a few select times for $\alpha=0.3$ and $\epsilon=1$; these values allow a direct comparison of Fig. 2d with Figs. $2 b$ and 2c. Such a comparison shows that the combined effects of nonlinearity and rotation are not a simple superposition of the individual effects due to each.

Comparing the nonlinear rotating case to its nonrotating counterpart (Fig. 2b), one notices three similarities: the nonlinearity breaks the right-left symmetry of the linear solution, a bore propagates to the right, and a rarefaction wave initially appears to the left. A similar comparison to the linear counterpart (Fig. 2c) shows some similarities as well: Poincaré waves tend to form 
due to the dispersive effects of rotation and the solution tends toward a sloping height field in geostrophic balance with a jet.

Several important features in Fig. 2d, however, result from the interplay of nonlinearity and rotation and cannot be seen in Figs. 2b and 2c. First, the amplitude of the initial discontinuity decays with time. ${ }^{3}$ Second, a new bore appears traveling to the left (see line marked $t=10$ in Fig. 2d), which will also decay with time (a third bore will form traveling to the right for large enough nonlinearity $\alpha$ ). This spontaneous appearance demonstrates that discontinuities form even in the presence of the dispersive effects of rotation (Houghton 1969; Williams and Hori 1970) and are not simply a result of our discontinuous initial condition (see also section 7). Third, the nonlinearity arrests some of the dispersive effects of rotation so that the length scale behind the left- and right-going discontinuity do not decrease with time (see Fig. 2c) as mentioned by Gill (1982).

Given that we have no analytical solutions at hand, it may be questioned whether our use of the term bore to describe the discontinuous-looking features in Fig. $2 \mathrm{~d}$ is appropriate. On the scale of the picture, it is not clear whether the features remain discontinuous or smooth out due to dispersion. In Fig. 4 we show five curves for the height field, for the case with $\alpha=.7$ at $t=20$, obtained by successively halving the spatial resolution. Notice from the scale on the abscissa that, by this time, what we call the initial right-going bore has decayed to roughly $2 \%$ of its original height (the third bore mentioned in the previous paragraph trails it) but that the discontinuity becomes sharper as the resolution is increased.

Another quantitative way of validating our claim that bores are present in nonlinear rotating adjustment is to use the fact that the speed and amplitude of bores are related by the Rankine-Hugoniot jump conditions; these are statements of mass and $x$-momentum conservation across shocks. In the nonrotating case, both the speed of the right-going bore $c_{s}$ and its amplitude are constant with time, whereas in the rotating case the amplitude decays. A corresponding change in speed should follow, as dictated by the jump conditions (Whitham 1974)

$$
\begin{array}{r}
{\left[(1+\alpha \eta)\left(\alpha u-c_{s}\right)\right]_{\text {back }}^{\text {front }}=0} \\
{\left[\alpha u(1+\alpha \eta)\left(\alpha u-c_{s}\right)+\frac{1}{2}(1+\alpha \eta)^{2}\right]_{\text {back }}^{\text {front }}=0 .}
\end{array}
$$

The brackets denote the difference, in the quantities within them, between the front and back of the discontinuities. Notice that these conditions do not depend on

\footnotetext{
${ }^{3}$ Bore decay has also been studied in the nonrotating context, the mechanism of the decay being a rarefaction wave catching up to a shock. See Friedrichs (1948) and Lax (1948).
}

rotation. The two relations above relate five quantities: $\eta_{b}, \eta_{f}, u_{b}, u_{f}, c_{s}$. Since the values of $u_{f}$ and $\eta_{\mathrm{f}}$ are known in the case of the initial right-going bore, one may use the two equations to derive a relation between any two of $\eta_{b}, u_{b}, c_{s}$.

In Fig. 5, we have plotted $\left(1+\alpha \eta_{b}\right) /\left(c_{r}\right)^{2}$ versus $c_{s} /$ $c_{r}\left[c_{r} \equiv(1-\alpha)^{1 / 2}\right]$ at equal increments in time, for three numerical calculations with different values of $\alpha$. Plotting these two quantities removes the $\alpha$ dependence of the curves and allows all the points to be drawn on the same plot. The solid line is the exact relationship as obtained from the Rankine-Hugoniot conditions (11) and (12). As time increases (see the arrow in Fig. 5), the amplitude of the bore decays and its speed decreases. The numerical values agree well with the relation obtained from the Rankine-Hugoniot conditions, confirming the idea that the regions of high gradients that appear in the nonlinear rotating adjustment are indeed bores.

Finally, it is interesting to illustrate the influence of rotation by drawing the characteristic lines in the $x-t$ plane; these lines are solutions of the two ordinary differential equations $d x / d t=\alpha u \pm(1+\alpha \eta)^{1 / 2}$. In Fig. $6 \mathrm{a}$, select characteristics for the classic dam-break problem are shown in dotted lines for the four regions mentioned in section $2 \mathrm{~b}$ (delimited with light solid lines); they are all straight lines and their crossing leads to the formation of a bore (heavy solid line). In Fig. 6b, we plot the corresponding characteristics for the rotating case. Notice the characteristics are curved and that this leads to more intersections and hence several bores, both left and right going. It is also important to note that, though all bores decay, some slow down while others speed up; this is readily seen from the curvature of their paths in the $x-t$ plane.

In summary, we emphasize that the combination of nonlinearity and rotation yields phenomena not present in the absence of either: the formation of multiple bores and their subsequent decay.

\section{Bore dependence on rotation and nonlinearity}

We have already mentioned how the parameter $\epsilon$, whose value is set by the strength of the rotation, can be set to 1 without loss of generality by scaling arguments on the governing dimensional equations. The physical implications of this are that for fixed $\alpha$, as rotation is increased, the total number of bores that forms remains unchanged, the bores decay faster, and the initial right-going bore slows down. The influence of the nonlinearity parameter $\alpha$, on the other hand, cannot be scaled away. We thus examine it next.

The behavior of adjustment as $\alpha$ varies can be seen in Fig. 7, where we have plotted the perturbation height field $\eta$ at four different times for $\alpha=0.1,0.4$, and 0.7 . Corresponding bores for each value of $\alpha$ decay faster (as a percentage of their original heights) as $\alpha$ is increased. Notice how the left-right asymmetry is more prominent for larger $\alpha$; compare, for instance, 
a linear, nonrotating

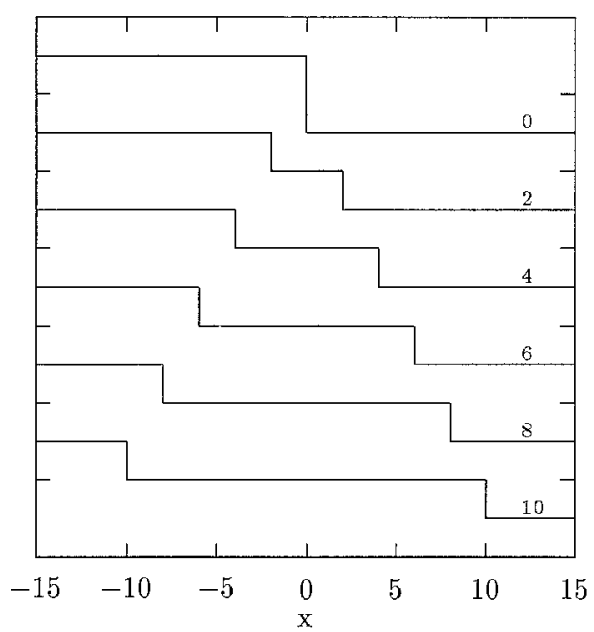

C linear, rotating

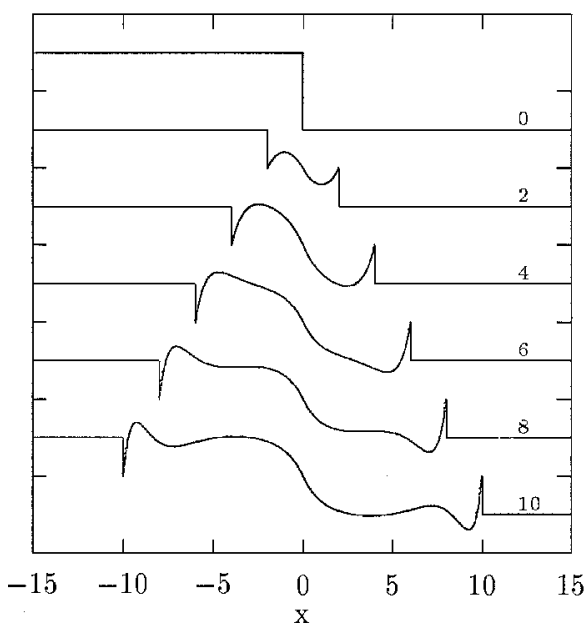

b nonlinear, nonrotating

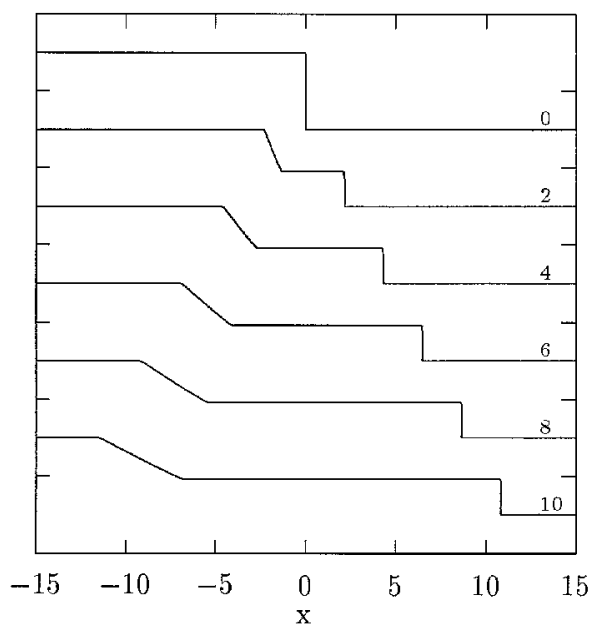

d nonlinear, rotating

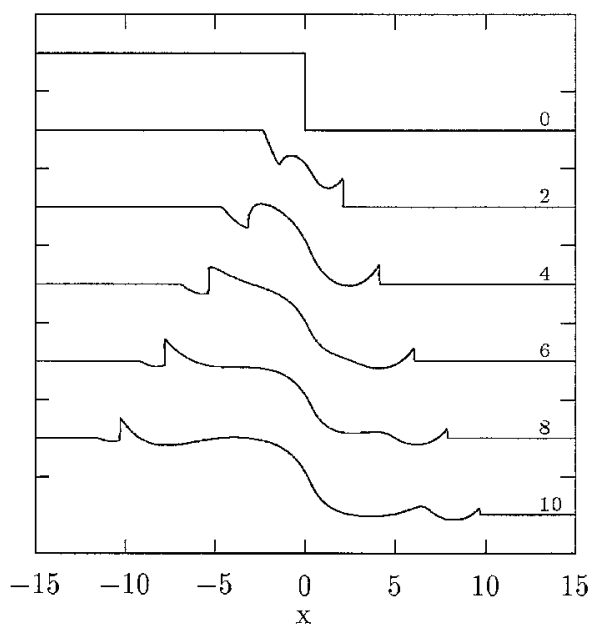

FIG. 2. The dimensionless height field $\eta$ given at six dimensionless times for the four cases: (a) linear, rotating; (b) nonlinear, nonrotating; (c) linear, rotating; and (d) nonlinear, rotating. Our results are presented in (d).

the curves for $\alpha=0.1$ and $\alpha=0.7$ at $t=8$ in Fig. 7. Recall that in the linear case, the solution is exactly symmetric (Gill 1976). Finally, the amplitudes of the Poincaré waves decay faster as the nonlinearity is increased.

The bore decay feature is clearly demonstrated in Fig. 8 where we plot the initial right-going bore amplitude versus time for the values of $\alpha$ in Fig. 7, as well as for a very nearly linear case with $\alpha=0.01$. In the latter case, since the initial height to the right of the discontinuity is approximately $98 \%$ of that to the left, one might expect the linear equations to hold. By and large they do, but notice that the bore still decays relatively rapidly-it is half of its original height at approximately $12 / f$-whereas it should remain constant according to the linear equations. This small discrepancy is related to the nonuniform validity of linearized theory as $t \rightarrow \infty$ (Whitham 1974). In the next section, we show that we can reproduce this decay effect by retaining nonlinear terms.

Plotting Fig. 8 on a log scale reveals no single power-law behavior for bore decay for the times considered here but interestingly reveals some independence from $\alpha$. Whitham's (1974) theory of the $1 / \sqrt{t}$ decay of the quantity $\log \left(\sqrt{1+\alpha \eta_{b}}-\sqrt{1+\alpha \eta_{f}}\right)$ was also not verified here unlike the results of Skamarock et al. (1996). We are unsure that the theory of Whitham's may be applied to the governing equations (1). 


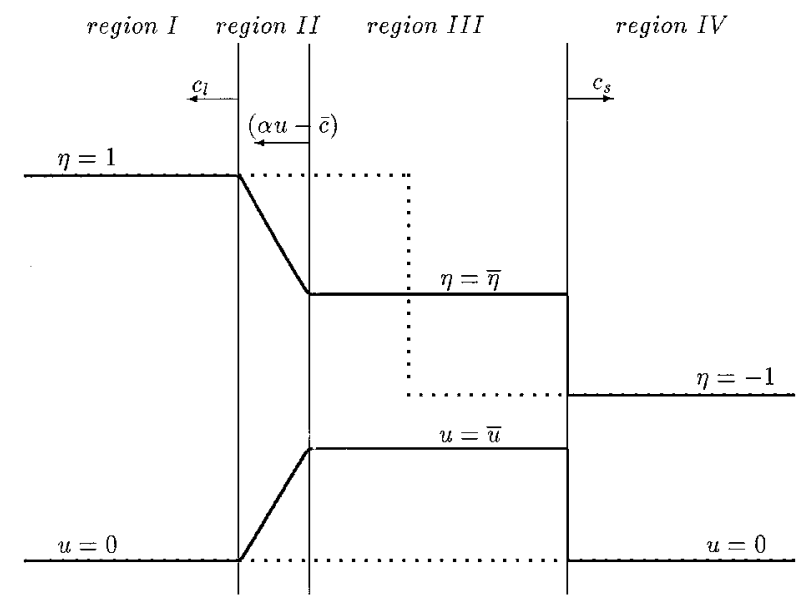

FIG. 3. Solution to the nonlinear, nonrotating (dam break) problem in dimensionless variables. Dotted lines indicate values at $t=0$. The bore speed $C_{s}, \bar{\eta}$, and $\bar{u}$ are given by the solution of (9).

\section{Asymptotic study in the weak rotational limit}

As we have shown, one obvious difference between nonlinear geostrophic adjustment and the nonrotating dam break problem is that the amplitude of the initial right-going bore decays in the presence of rotation. In order to understand this phenomenon, we have carried out an asymptotic analysis of the shallow-water equations (4). A similar analysis for the weak nonlinear regime $(\alpha \ll 1)$ was performed by Blumen (1967b). Here we are interested in the complementary regime of weak rotation, that is, $\epsilon \ll 1$, and given that the fully nonlinear solution to the dam break problem is known (Stoker 1958), we let $\alpha=O(1)$. The small $\epsilon$ regime may be interpreted physically as meaning weak rotational effects since $\epsilon \equiv f L / \sqrt{g H}$ and we may take $f \ll \sqrt{g H} /$ $L$. Note that the choice of $\epsilon \ll 1$ implies that validity of the asymptotic solution is restricted to $t \ll \epsilon^{-1}$ (dimensionally, $\left.t \ll f^{-1}\right)$. However, beyond providing a further check of the numerics, the asymptotic analysis is useful in that it yields an analytic expression for the time decay of the initial right-going bore amplitude due to rotation. Once the time decay is shown for any nonzero rotation, one then only needs to invoke similarity to demonstrate that increasing the rotation increases the rate of the initial right-going bore decay.

The technical details of the asymptotic expansion are given in appendix B. Here we only present the key steps of the derivation and physically interpret the results. With $\epsilon \ll 1$ and $\alpha=O(1)$, we expand the solutions of Eq. (4) with initial conditions (5) in the usual form:

$$
\begin{aligned}
& u(x, t)=u^{(0)}(x, t)+\epsilon u^{(1)}(x, t)+\epsilon^{2} u^{(2)}(x, t)+\cdots \\
& v(x, t)=v^{(0)}(x, t)+\epsilon v^{(1)}(x, t)+\epsilon^{2} v^{(2)}(x, t)+\cdots \\
& \eta(x, t)=\eta^{(0)}(x, t)+\epsilon \eta^{(1)}(x, t)+\epsilon^{2} \eta^{(2)}(x, t)+\cdots .
\end{aligned}
$$

We then substitute the above into Eq. (4) and collect the different orders in $\epsilon$.

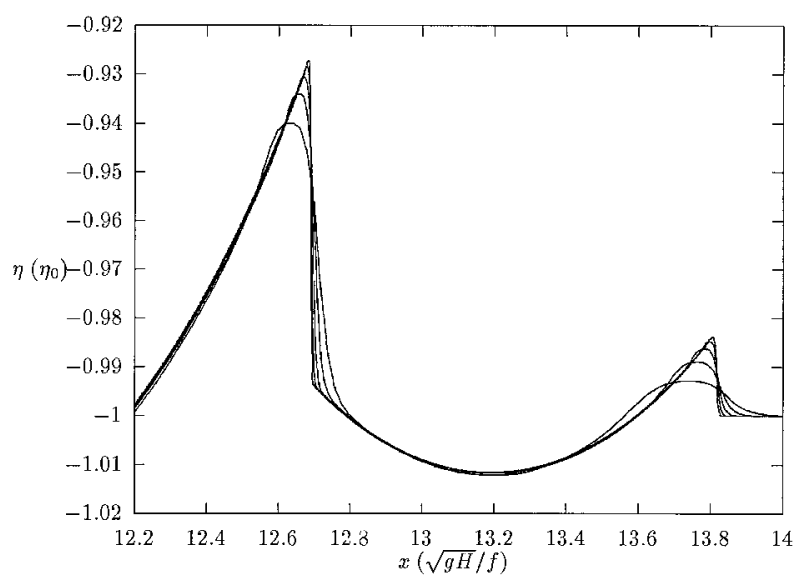

FIG. 4. As the spatial resolution is successively halved from $\Delta x=$ .02 to $\Delta x=.000125$, it becomes clearer that as bores decay, they tend to remain discontinuous rather than dispersing away. Plot is taken at $t=20(1 / f)$ when the initial right-going bore has decayed to $2 \%$ of its original height. A secondary bore trails the first.

At $O(1)$ the nonrotating dam break problem is recovered; hence $u^{(0)}, v^{(0)}$, and $\eta^{(0)}$ are given by (8). Notice that $v=0$ at lowest order; the rotational effects generating $v$ come in at $O(\epsilon)$, as pointed out by Houghton (1969).

At $O(\epsilon), u^{(1)}=\eta^{(1)}=0$ while $v^{(1)}$ is generated from the equation

$$
\frac{\partial v^{(1)}}{\partial t}+\alpha\left(u^{(0)} \frac{\partial v^{(1)}}{\partial x}\right)=-u^{(0)} .
$$

Hence, the first-order rotational effect is the formation of the $v$ velocity, which remains zero at all orders in the dam-break problem. The solution for $\boldsymbol{v}^{(1)}$ is given in appendix B.

At $O\left(\epsilon^{2}\right)$ and in region III (cf. Fig. 6), $v^{(2)}=0$ and $u^{(2)}$ and $\eta^{(2)}$ satisfy

$$
\begin{aligned}
\frac{\partial u^{(2)}}{\partial t}+\alpha\left(u^{(0)} \frac{\partial u^{(2)}}{\partial x}\right)+\frac{\partial \eta^{(2)}}{\partial x} & =v^{(1)} \\
\frac{\partial \eta^{(2)}}{\partial t}+\alpha u^{(0)} \frac{\partial \eta^{(2)}}{\partial x}+\left(1+\alpha \eta^{(0)}\right) \frac{\partial u^{(2)}}{\partial x} & =0 .
\end{aligned}
$$

This nonhomogeneous linear system shows how $u^{(2)}$ and $\eta^{(2)}$ are generated from $v^{(1)}$; the cumbersome analytic expressions for the solutions at $O\left(\epsilon^{2}\right)$ are given in appendix B.

For the case $\alpha=0.3$, the asymptotic solution for the first three orders of perturbation in $\epsilon$ is plotted in Fig. 9 for three different times of the evolution; the solid lines are the solutions obtained from the numerical integration and the dotted lines represent the above asymptotic solution. The agreement is rather good. The generation of a transverse velocity $v$ by the rotation at $O(\epsilon)$ (Fig. 9b) causes a decrease in the velocity $u$ at $O\left(\epsilon^{2}\right)$ (Fig. 9a) with an associated decrease in the amplitude of the right-going bore at the same order (Fig. 

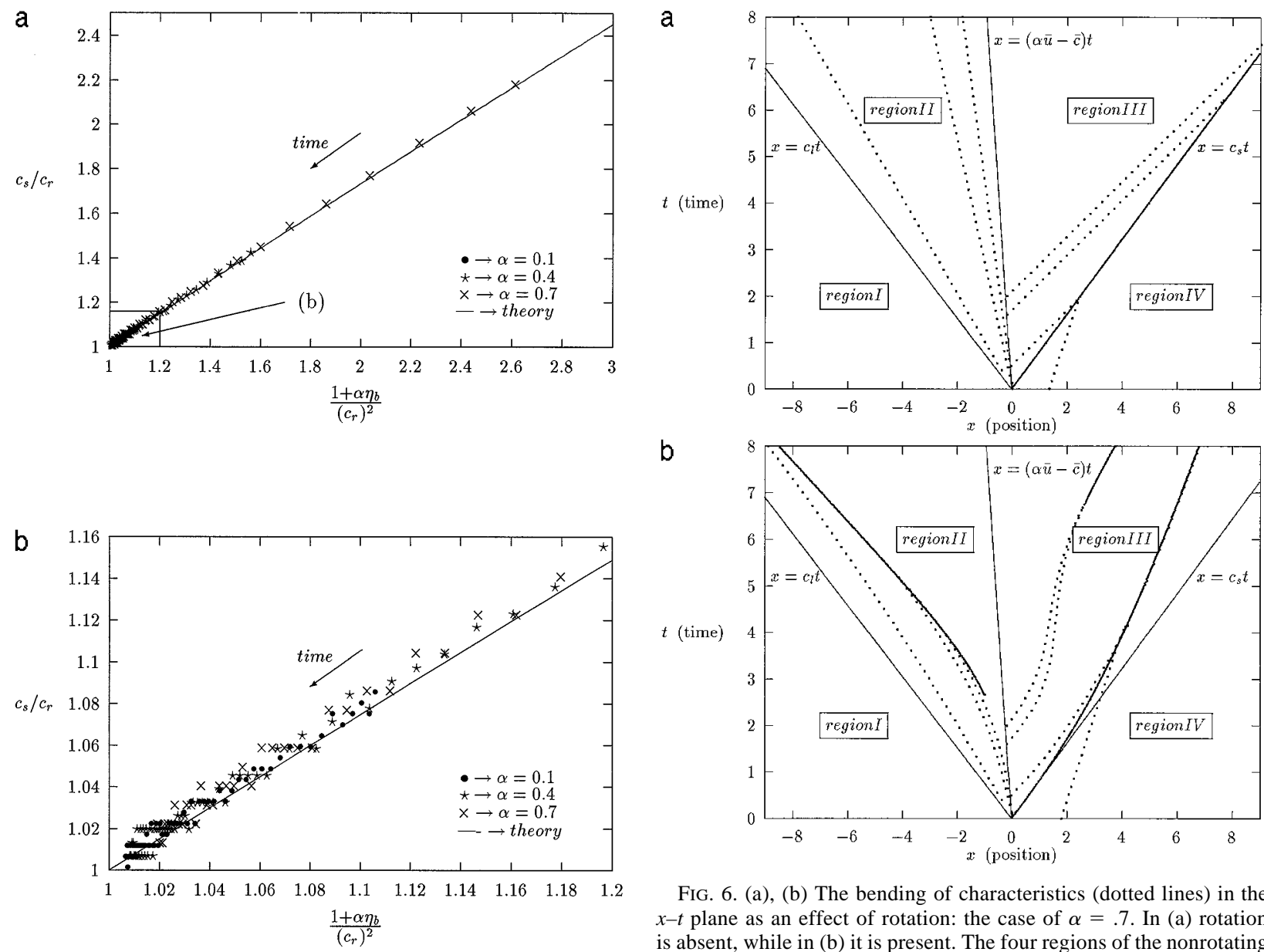

FIG. 5. (a), (b) The theoretical Rankine-Hugoniot relationship between the right-going bore height $\eta_{b}$ and its speed $c_{s}$ plotted in solid lines. The numerical values of the same quantities obtained for three cases of $\alpha$ at equal increments in time are also plotted in the figure. Errors are \pm .050 for $\alpha=.1, \pm .045$ for $\alpha=.4$, and \pm .046 for $\alpha=$ .7 The case $\alpha=.01$ was omitted due to clutter but agreed within error bounds to the theoretical prediction. The implication is that the right-going entity is in fact a bore. (b) An enlargement of the lower left corner of (a).

9c). Notice that the agreement between the asymptotic solution and the computation worsens as time increases; this is due to the breakdown of the asymptotics past $t$ $=\epsilon^{-1}$.

From the above asymptotic analysis, it appears that the formation of the transverse $v$ velocity plays a crucial role in bore decay. For waves traveling along a coastline, the velocity perpendicular to the coastline (the analog of the $v$ velocity in our problem) must be weak because of the boundary condition requiring no normal flow. Thus, bore decay might not be as pronounced in that case, a result that Skamarock et al. (1996) seem to show. How much bore decay exists in a less restrictive fully two-dimensional setting remains to be seen.

The asymptotic solution is also able to capture the already mentioned feature of section 4 that greater nonlinearity $\alpha$ leads to faster bore decay. From the expres-

FIG. 6. (a), (b) The bending of characteristics (dotted lines) in the $x-t$ plane as an effect of rotation: the case of $\alpha=$.7. In (a) rotation is absent, while in (b) it is present. The four regions of the nonrotating case (a) are also plotted in (b) to facilitate comparison and are marked off by the light solid lines. Intersection of characteristic lines implies the presence of a bore. Bore paths are given in dark, solid lines.

sions in appendix $\mathrm{B}$, the amplitude of the right-going bore $\eta_{b}$ as a function of time is found to be of the form

$$
\eta_{b}(t)=\bar{\eta}-\epsilon^{2} K(\alpha) t^{2}+O\left(\epsilon^{4}\right)
$$

with

$$
\begin{aligned}
K(\alpha) \equiv & \frac{1}{2 \alpha \bar{c}}\left(\alpha \bar{u}-c_{s}+\bar{c}\right)^{2} \\
& \times\left[\frac{1}{216 c_{l}^{2}}\left(-\alpha \bar{u}+2 c_{l}+\bar{c}\right)^{3}+\frac{3}{8} \alpha \bar{u}-\frac{1}{8} \bar{c}\right] \\
& -\frac{\bar{u}}{4 \bar{c}}\left(\alpha \bar{u}-c_{s}\right)\left(\alpha \bar{u}-c_{s}+\bar{c}\right),
\end{aligned}
$$

where $\bar{u}, \bar{\eta}, c_{s}$ are obtained from the dam-break solution (8) and $\bar{c} \equiv \sqrt{1+\alpha \bar{\eta}}$. Since $K(\alpha)$ is positive and increases with $\alpha$, Eq. (17) shows that the right-going bore amplitude decreases with time and the decay is faster for greater $\alpha$. The numerics agree extremely well with this formula. 

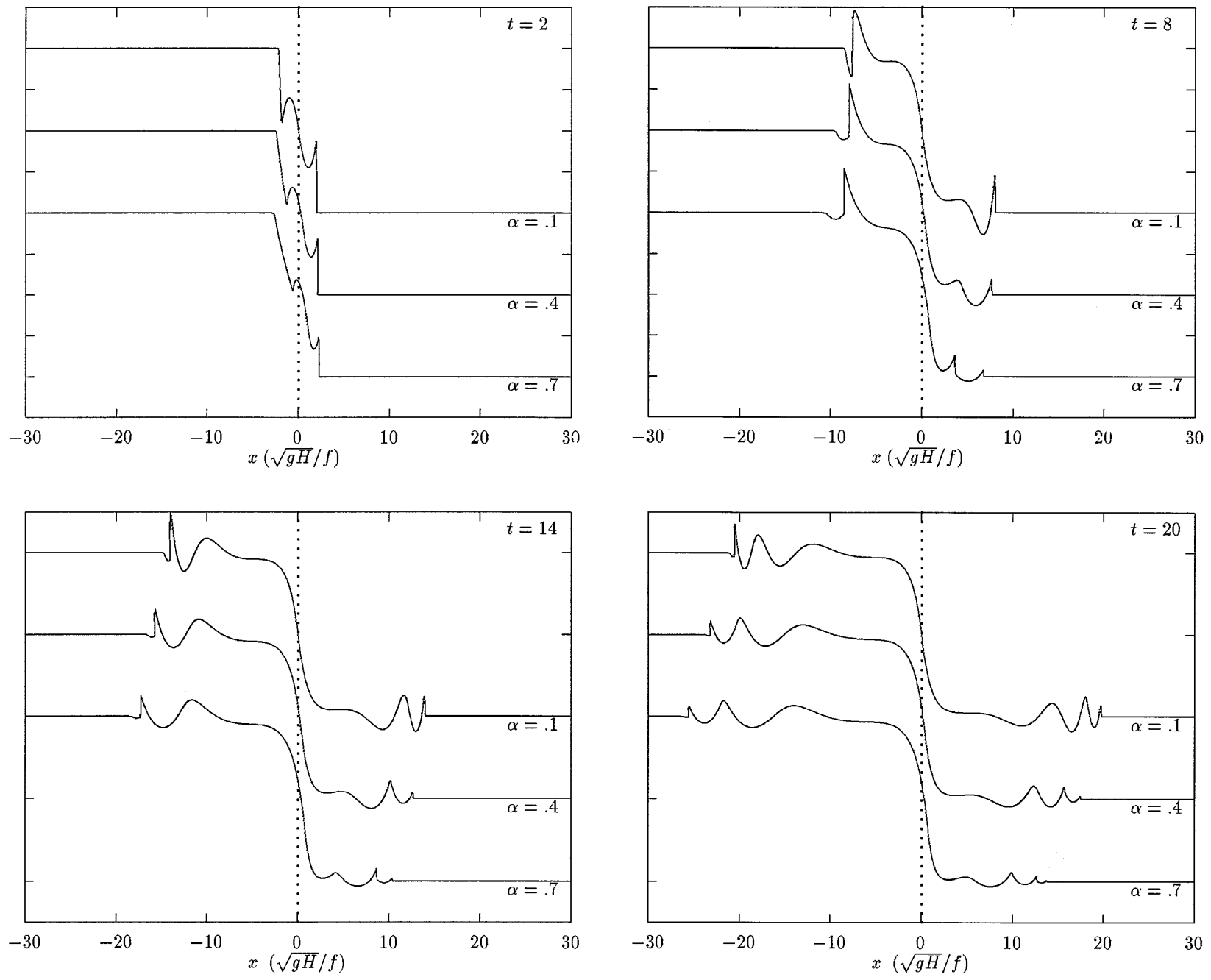

FIG. 7. Effect of nonlinearity on adjustment: an increase in the nonlinearity leads to increased asymmetry, increased net shift to the right, and increased rate of bore decay.

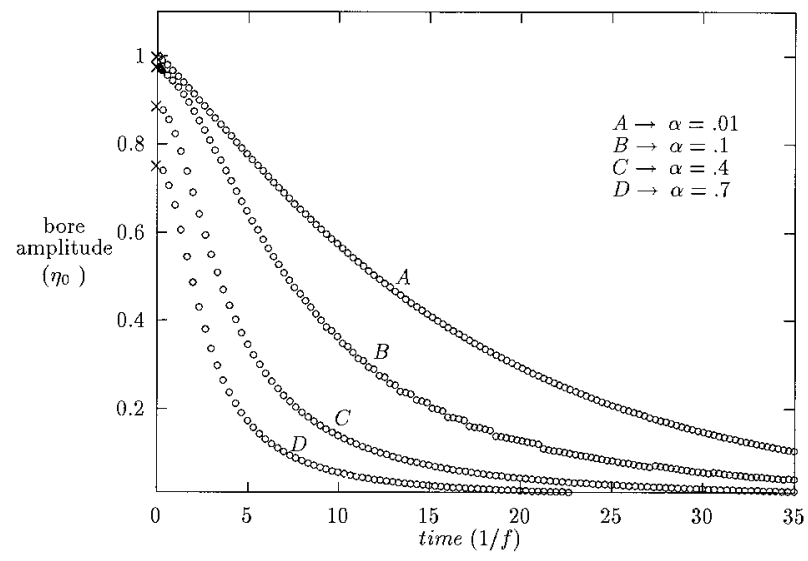

FIG. 8. The initial right-going bore height as a function of time for four cases of $\alpha$ in the rotating problem. The $\times$ 's at $t=0^{+}$denote the values of the corresponding (constant) bore heights in the nonrotating problem.

\section{The long-time approach to the balanced state}

In this section, we present some results of long-time numerical integrations, which we have performed in an attempt to answer the following questions:

- How long does it take for adjustment to become complete, and how does this time depend on the nonlinearity parameter $\alpha$ ?

- How well does the end state predicted using conservation methods match with the one that emerges from the initial value problem?

We cannot answer these two questions in a definite way since we can only integrate out to a finite time. However, long time integrations show a clear tendency for decaying oscillations, implying that a balanced state is being approached. We start in section 6a by addressing some delicate issues pertaining to small (though inevitable) numerical errors and how they might be affecting our long-time results. In section $6 \mathrm{~b}$ we study the time- 

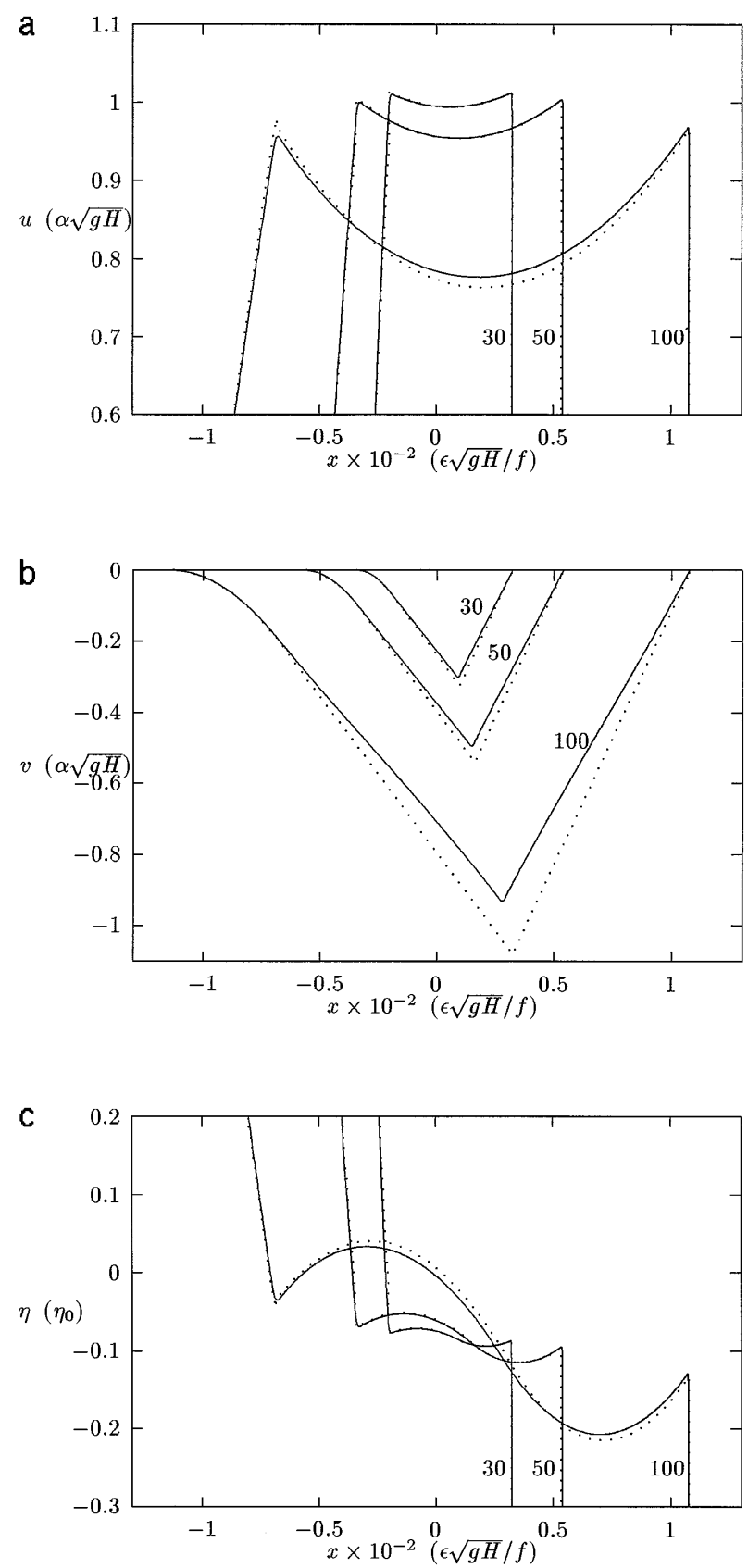

FIG. 9. Dam-break problem perturbed by weak rotation $(\epsilon=0.01)$ for the case $\alpha=0.3$. The asymptotic solution is plotted in dotted lines and the numerical solution is plotted in solid lines. Numbers next to the curves indicate time. Both solutions exhibit bore decay.

scale of adjustment, and in section $6 \mathrm{c}$ we examine the end states.

\section{a. The long-time accumulation of numerical errors}

It should be obvious that however accurate a numerical scheme, computational errors are bound to accumulate when a numerical integration is carried out to

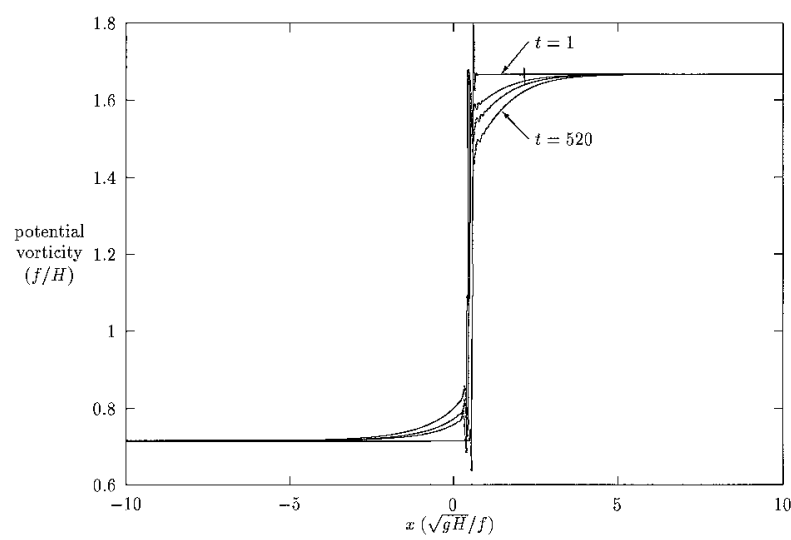

FIG. 10. Numerical degradation in time of PV near the front for a typical case: $\alpha=0.4$. The theoretical solution is a step function advecting back and forth in time. The PV is plotted at $t=1,226$, $330,520(1 / f)$. The large overshoot on the $t=1$ curve is a result of the discontinuous $x$ derivative in the $v$-velocity jet. The signature of the bore is also visible as a blip on this curve.

very long times (in our case, we have integrated out to several hundred $1 / f$ encompassing over 430000 time steps). One concern has to do with the reflection of Poincaré waves at the edges of the finite computational domain. We eliminate the possibility of such waves affecting our results by using a very large $\left(700 L_{d}\right.$ wide) domain; the adjustment region, which we will be concerned with, is at most $20 L_{d}$ wide centered at the origin. Furthermore, even though we have implemented the radiative boundary conditions proposed by Orlanski (1976), we stop the integrations before any reflected waves have a chance of entering our region of interest. As a measure of the accuracy of our calculations, we note that before the waves hit the computational domain boundaries, mass is conserved to at least eight decimal places, though this is not surprising since schemes in conservation-law form are designed to do this.

We have found, however, that numerical dissipation becomes apparent when long time integrations are performed. The PV field provides a rigorous test of dissipative effects. The initial PV distribution is a step function and, since PV is materially advected, its gradients should remain infinitely steep. The typical degradation present in our long time integrations is shown in Fig. 10, where the PV is plotted at selected times over the adjustment region of interest; we use a resolution of 200 grid points per $L_{d}$, so that 4000 grid points are represented in that figure. Notice how the width of the front remains constant, while the degradation occurs at the corners of the initial step. The overshoot is an effect of the discontinuity in the derivative of $v$. Since we have found that increasing the spatial and time resolution makes the PV front sharper, the question is really one of resolution rather than a limitation of the numerical scheme. Even so, we feel that the conclusions we draw below do not depend on the numerical effects 
discussed here since these effects only seem to add a very slight drift in time to our solutions.

\section{b. The timescale of adjustment and its dependence on nonlinearity}

The timescale associated with adjustment is short; in the words of Killworth (1992), "adjustment problems are characterized by rapid modification, on the inertial time scale ...". This fact seems to have lead to a widespread belief that the time required to complete the adjustment process is also relatively short, say on the order of a few inertial periods (Obukhov 1949). But, after investigating the effects of the firstorder nonlinear corrections to a linear solution, Blumen (1967b) argued that nonlinearity might lead to an increase in the timescale of adjustment. In this paper we integrate the fully nonlinear system and are thus in a position to reexamine that possibility. To answer the question of "how long does the adjustment process take?" one must decide what measure is to be used to determine when adjustment reaches completion. It turns out that the answer depends rather sensitively on the measure chosen, and thus it is difficult to give an answer in general. We will thus limit ourselves to describing the convergence properties of three distinct, meaningful measures: a pointwise measure, an integral measure, and an energy measure. In each case we explore the four values $\alpha=0,0.1,0.4$, and 0.7 to examine the effects of nonlinearity.

We start with a simple pointwise measure. In Fig. 11 we plot the three fields $u, v$, and $\eta$ at the origin over a time span of $520(1 / f)$. The first surprising result is that the influence of $\alpha$ is felt quite strongly in the $v$ and $\eta$ fields, but seems to be nonexistent on the $u$ field (there are actually four superimposed lines in the $u$ plot). Moreover, a comparison of the $v$ and $\eta$ plots yield conflicting results: from the $\eta$ field it would seem that adjustment is slower if the system is more nonlinear (Blumen 1967b), whereas the $v$ field indicates the contrary. Furthermore, had we shown a point different from the origin, the results would be different. So it should be clear that pointwise measure are not robust; the underlying reason for this is the greater nonlinear shifting that takes place as $\alpha$ increases.

The second measure we consider-the root-meansquare averaged (rms) values of $u, v$, and $h$ over a fixed region-was chosen to remove some of the problems associated with the previous measure, but it exhibits some dependence on the size of the region of averaging. In practice however, one wants to pick averaging regions reasonably near the PV front (i.e., the geostrophically adjusted jet), so we have examined regions 10 and $20 L_{d}$ wide centered at the origin.

The results for a $10 L_{d}$ wide region are plotted in Fig. 12 (those for a $20 L_{d}$ wide region are similar). Notice that with this rms measure, the height field $\eta$ adjusts more quickly than the velocity fields. This turns out to be robust behavior (as will be demonstrated later in Fig. 15) and clearly illustrates how the adjustment time is fundamentally dependent on which individual variable is measured. The height field essentially adjusts within 50 to $1001 / f$ (notice the small drift caused by the numerical dissipation mentioned in the previous section); the velocity fields, on the contrary, exhibit substantial amplitude oscillation, up to order of $20 \%$ of their final value, even after many hundred $1 / f$. For the velocity fields, the amplitude of the oscillations decreases faster as $\alpha$ increases, though this effect is barely discernable from the figure.

As a final measure of adjustment, we consider the well-known ratio

$$
\left|\frac{\Delta \mathrm{KE}}{\Delta \mathrm{PE}}\right|
$$

in a fixed region around the origin. This choice eliminates the difficulty of choosing between the $u, v$, and $\eta$ variables as adjustment measures by combining them into a single quantity. Additionally, this choice is relatively insensitive to the size of the region in which the energies are computed. We examined regions 10 and $20 L_{d}$ wide, but present results for the $20 L_{d}$ case in an attempt to better match the theoretically predicted end-state energy ratio of $1 / 3$ (Gill 1976; Middleton 1987; Boss and Thompson 1995).

In Fig. 13, we plot the energy ratios for all four cases of $\alpha$. Because this energy measure is particularly sensitive to the numerical dissipation discussed earlier, the downward trends in $\triangle \mathrm{KE}$ and $\triangle \mathrm{PE}$ have been subtracted off before forming the ratio (this does not change the relative oscillation magnitudes of either $\Delta \mathrm{KE}$ or $\Delta \mathrm{PE}$ ). We believe that because of this numerical problem, and not due to the presence of bores, the ratio does not precisely oscillate about $1 / 3$. The decay rate is initially high (highest for small $\alpha$ ) but soon tapers off. The key point here is that the peakto-peak oscillation amplitude is as large as $30 \%$ of the final value for all $\alpha$, even after several hundred $1 / f$. In this sense, one might say that the energy ratio takes a "long" time to converge to the " $1 / 3$ " value. As $\alpha$ increases, the oscillations are slightly smaller in magnitude at a given time beyond 25(1/f).

In summary, though it is clear that the adjustment time is highly dependent on the chosen measure, one robust result emerges from the above computations: the adjustment seems to proceed in two distinct phases. The rate of decay of the amplitude oscillations is initially very high, but after about $50(1 / f)$ or so it severely slows down, so that even after several hundred $1 / f$, substantial inertial oscillations persist in either the $u$ and $v$ fields or the energy ratio

$$
\left|\frac{\Delta \mathrm{KE}}{\Delta \mathrm{PE}}\right|
$$



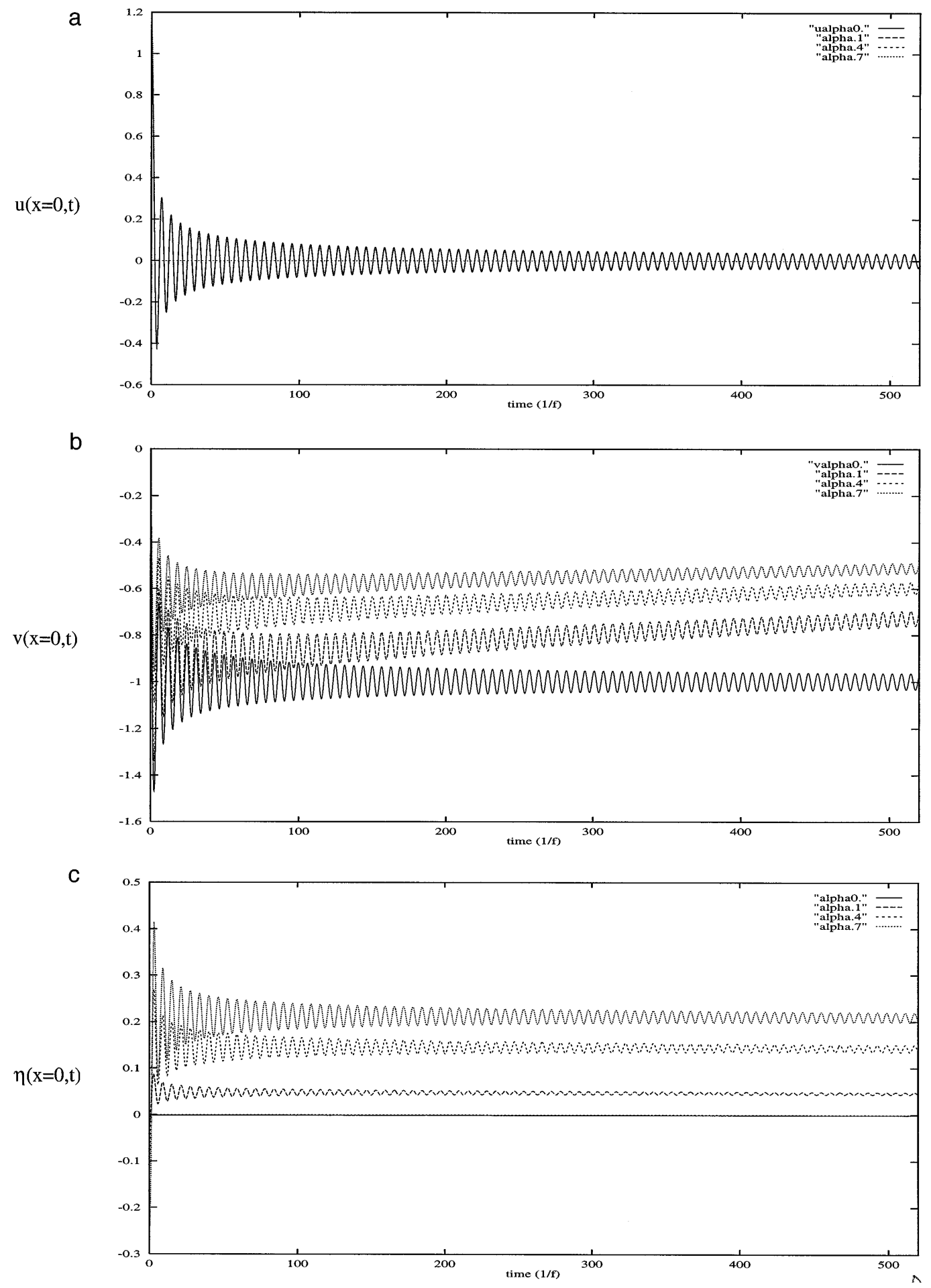

FIG. 11. Pointwise values of $u, v$, and $\eta$ at the origin versus time, each plotted for four cases of nonlinearity $\alpha$. Here $\eta$ and $v$ display a dependence on nonlinearity, while $u$ does not. Hence, pointwise measures may give a misleading measure of adjustment time. 
a
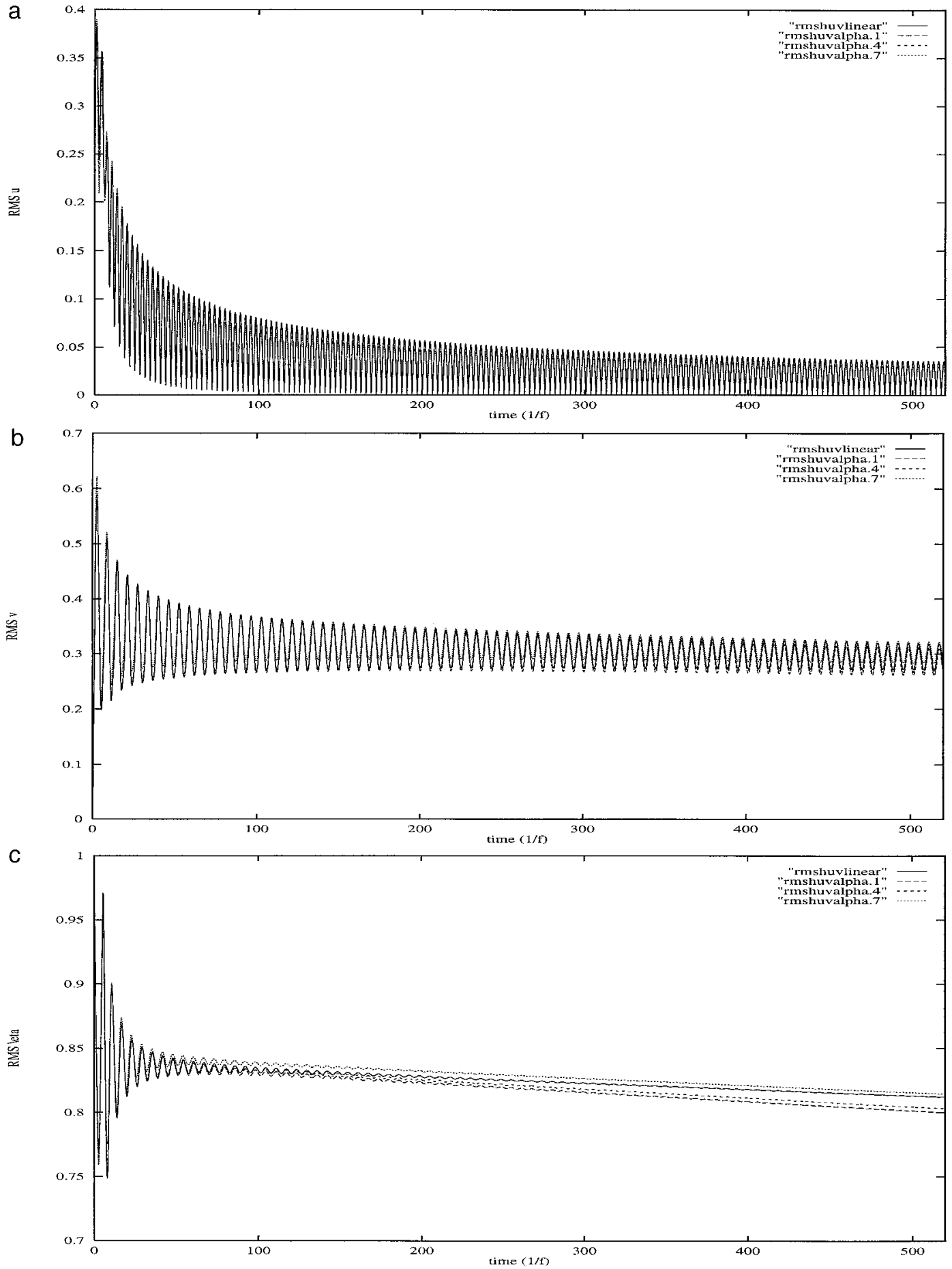

FIG. 12. Rms averages of $u, v$, and $\eta$ versus time in a region $10 L_{d}$ wide centered at the origin. Each is plotted for four cases of nonlinearity $\alpha$. The correct sense that the velocity fields take considerably longer to adjust than the height field is obtained using this measure. The $u$ rms average appears, but does not, oscillate at double the inertial frequency. This is because its mean value is zero, unlike $\eta$ and $v$.

(after the rapid decay period, the height field, though, equilibrates much faster). Whether these long time oscillations are of practical interest is unclear because there is reason to believe that adjustment in two-di- mensions is faster than in one (Blumen 1972). However, since they persist for such very long times, the common neglect of dissipation in (time independent) end-state adjustment studies could perhaps be reexamined. 

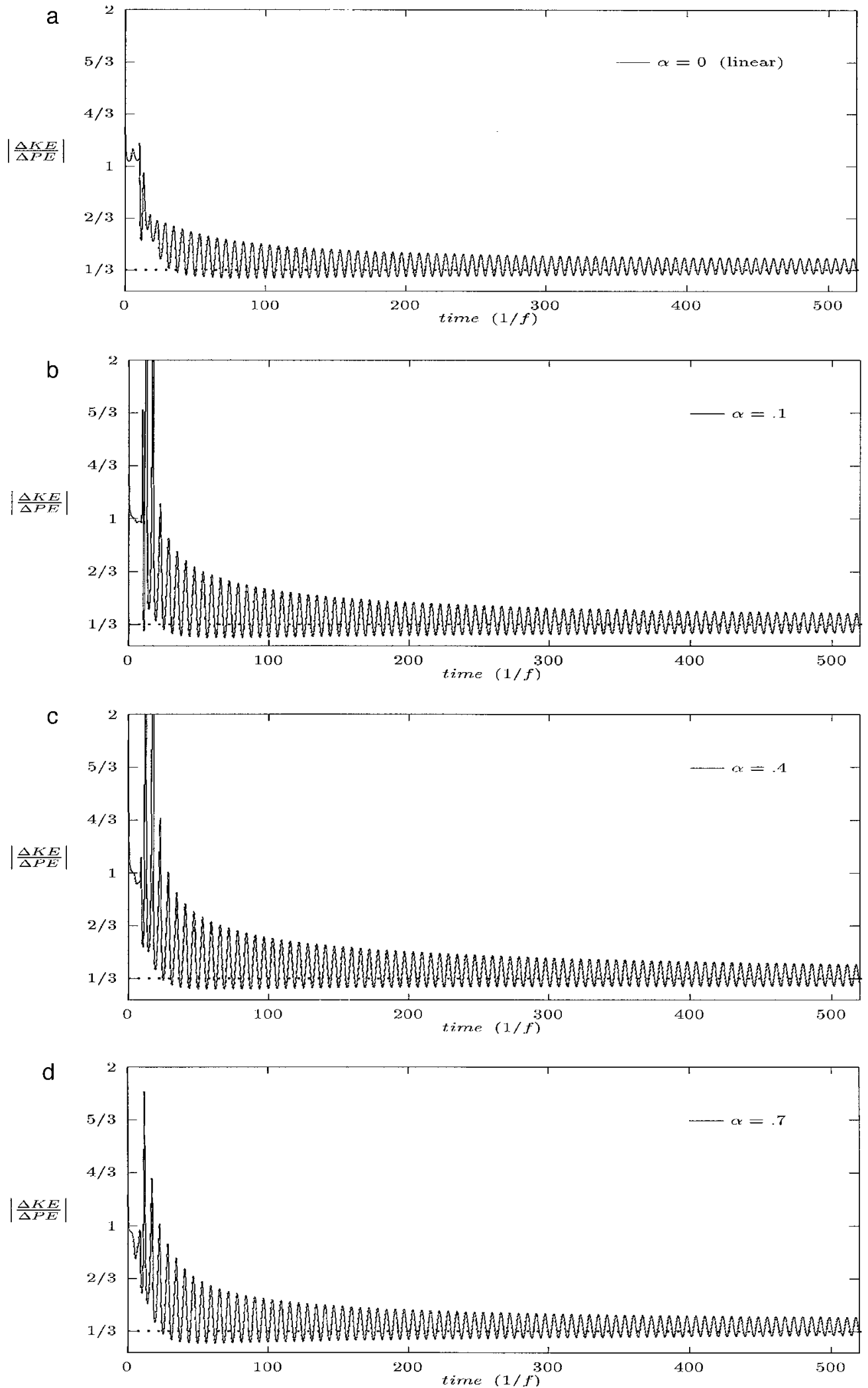

FIG. 13. Energy ratio $|\Delta \mathrm{KE} / \Delta \mathrm{PE}|$ versus time for four cases of $\alpha$ : (a) 0 , (b) 0.1 , (c) 0.4 , and (d) 0.7 for a region $20 L_{d}$ wide centered at the origin. The oscillations have peak to peak amplitudes of roughly $30 \%$ of their final values at the end of the integration. 


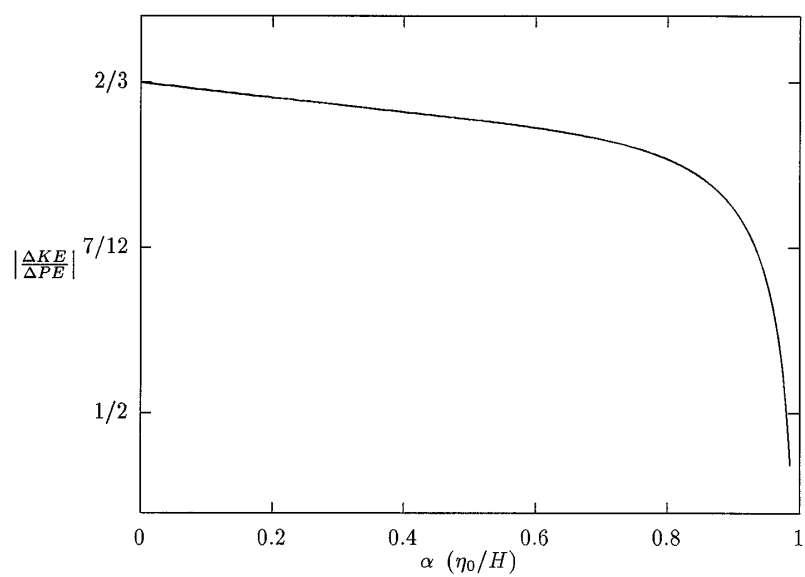

FIG. 14. End state energy ratios for the (nonrotating) dam-break problem as a function of $\alpha$. In contrast to the rotating case, the ratio displays $\alpha$ dependence.

\section{c. End states: Theory versus numerics}

Before comparing the final state predicted by conservation methods to that obtained by the numerical initial value problem, we briefly motivate why a discrepancy between the two might be expected. Since we have shown that bores form, one expects that energy will be dissipated during adjustment. Because of this, it is not clear that PV conservation, used in the calculation of the final state, is a valid assumption (see also Pratt 1983). Hence, it is conceivable that the final state predicted using conservation principles may be quite different from the one reached by a time-evolving system.

Stoker (1958) derived an expression for the total energy loss due to a bore as a function of the height difference across it. The nondimensional version of his expression is

$$
\frac{D E}{D t}=\frac{c_{s} \alpha^{3}\left(\eta_{f}-\eta_{b}\right)^{3}}{2\left(1+\alpha \eta_{b}\right)},
$$

where $\eta_{f}$ and $\eta_{b}$ denote the heights in front of and in back of the bore respectively, $c_{s}$ is the bore speed, and the nondimensional energy $E$, the sum of kinetic and potential parts, is given by

$$
E=\int\left[\frac{1}{2} \alpha^{2}(1+\alpha \eta)\left(u^{2}\right)+\frac{1}{2}(1+\alpha \eta)^{2}\right] d x .
$$

Notice that in the linear case as $\alpha \rightarrow 0$, the energy loss vanishes, even in the presence of discontinuities.

Before discussing the rotating case, we illustrate in Fig. 14 the final state ratio

$$
\left|\frac{\Delta \mathrm{KE}}{\Delta \mathrm{PE}}\right|
$$

for the nonrotating solution (8) of the dam-break problem. Notice that it strongly depends on the nonlinearity

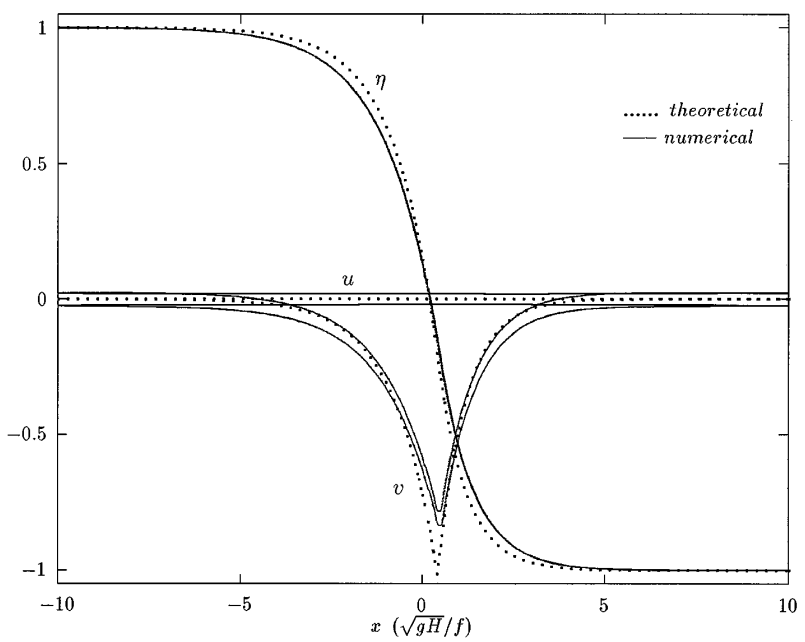

FIG. 15. The long-time numerically calculated height and velocity fields (solid lines) plotted at $t=517$ and $t=520(1 / f)$ corresponding to the minimum and maximum oscillations at the end of the integration. The dotted lines represent the theoretical solution.

$\alpha$. This is in stark contrast to the results of Boss and Thomson (1995) who have shown that this ratio is independent of $\alpha$ when rotation is present.

As we have already pointed out in our numerical solutions, the $u$ and $v$ fields exhibit oscillations even after several hundred 1/f; therefore, we cannot compare our results to those of Boss and Thompson at a single point in time since we never attain a "final state." In Fig. 15, we superimpose the Boss and Thomson solution onto our field at $t=517 / f$ and $t=520 / f$ corresponding to the minimum and maximum of the oscillation, for the representative case $\alpha=0.4$. The agreement is surprisingly good. Note that the numerical dissipation is responsible for the fact that the numerically computed jet in $v$ has a slightly smaller amplitude (about 80\%) than the predicted one and that the height field is much better adjusted than the $u$ and $v$ fields.

This somewhat surprising agreement between the theory and the initial value problem in the presence of bores may be easily understood. In Fig. 16, we plot as a function of time the total energy $E$ in a region $20 L_{d}$ wide centered at the origin for both the rotating (solid) and the corresponding nonrotating (dotted) cases. The energy is normalized to 1 at $t=0$ for each of the three cases of $\alpha: \alpha=0.1,0.4$, and 0.7. No wave flux of energy occurs out of the region for these times; in the absence of bores, therefore, the energy should be constant. The constant slope of the dotted lines represents the constant energy loss due to bores in the absence of rotation. Note how the corresponding rotating curves flatten out as time progresses, indicating that the energy loss in the presence of rotation is severely reduced (and eventually arrested). The reason for this is simple: the bores decay away! In a nutshell then, the results here suggests that though bores may have potential effects 


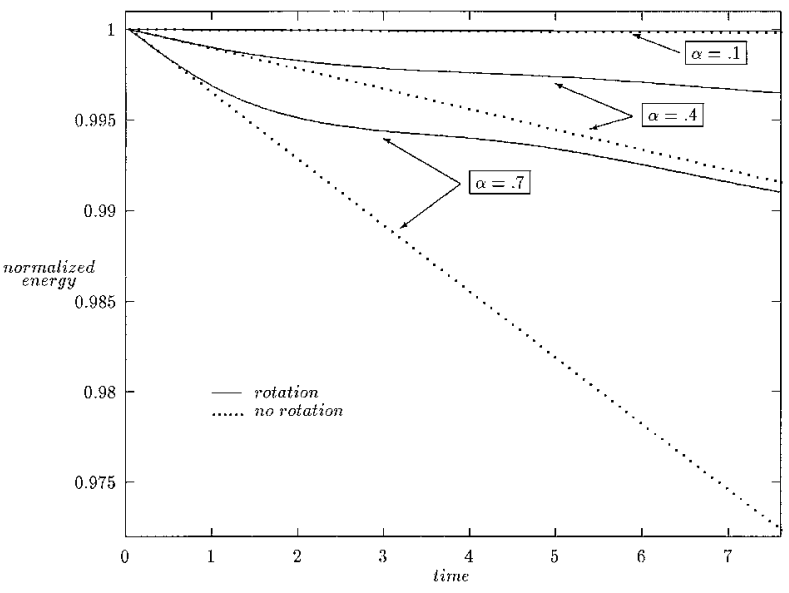

FIG. 16. Nondimensional energy $E$ in a region $-10<x<10$ wide for the rotating and nonrotating nonlinear problems. The key feature is that energy loss tapers off and is eventually arrested when rotation present.

on the long-time solution, because they decay, their effects are negligible.

\section{Bore formation from smooth initial conditions}

In this section, our aim is to demonstrate that the presence of bores in the adjustment problem is not a mere consequence of the discontinuous initial conditions used in the previous sections. We thus consider an initial height profile in the shape of hyperbolic tangent and investigate how steep the initial profile must be in order for bores to develop.

Again solving Eqs. (4) with $\alpha \neq 0$ and $\epsilon=1$, we take the $u$ and $v$ velocities to be initially zero and the dimensional initial height to be

$$
h(x, t=0)=h_{l}-\frac{\alpha}{2} H\left[1+\tanh \left(\frac{x}{L_{0}}\right)\right],
$$

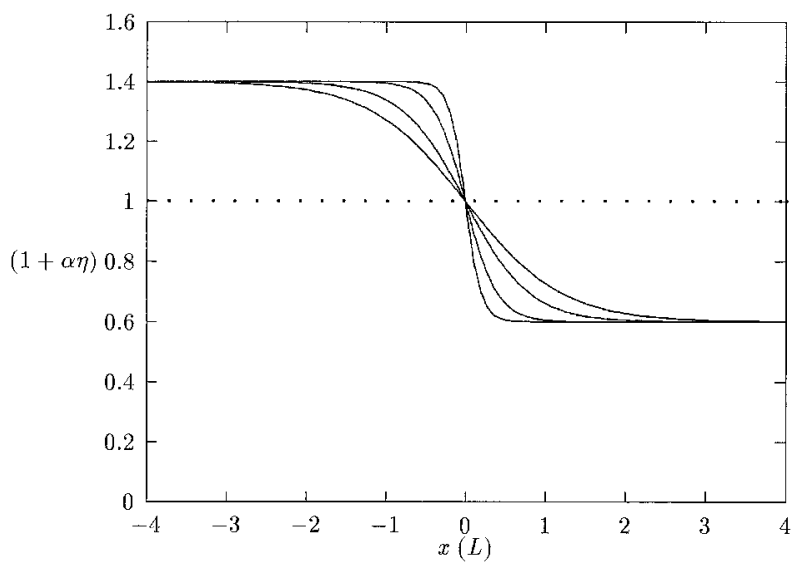

FIG. 17. Initial tanh-shaped height profiles with $\alpha=.4$ for four cases of $\kappa \equiv 2 L / \alpha$. From most steep to least steep, $\kappa=1,2,4,6$.

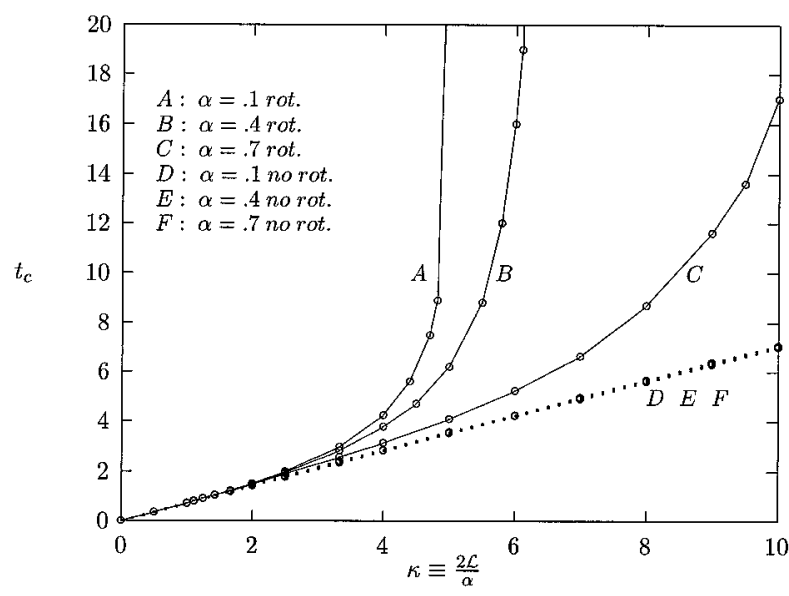

FIG. 18. Critical time $\left(t_{c}\right)$ for onset of bore formation as a function of the smoothness of an initial tanh height profile, measured by $\kappa$ (larger $\kappa$ implies smoother initial profile) and plotted in solid lines. The equivalent nonrotating cases are plotted in dotted lines. $t_{c}=\infty$ for line $C$ at $\kappa=5$ and at $\kappa=6.2$ for line $B$.

which translates into the nondimensional initial condition using (3) and dropping primes,

$$
\eta(x, t=0)=-\tanh \left(\frac{x}{\mathcal{L}}\right) .
$$

The parameter $\mathcal{L} \equiv L_{0} / L$ could be used as a dimensionless steepness parameter. However, notice from Eq. (20) that for fixed $\mathcal{L}$, the steepness changes as the nonlinearity parameter $\alpha$ is varied. The proper steepness parameter must be a measure of the slope of the height profile at $x=0$ since the slope determines when characteristics first cross and thus when bores first form. We have chosen to quantify initial steepness with the parameter $\kappa \equiv(2 \mathcal{L} / \alpha)$, which is the reciprocal of the slope of the dimensional height field at the origin at $t=0$. Constant $\kappa$ preserves the aspect ratio and (roughly) the slope as $\alpha$ changes. The initial height profiles for $\alpha=0.4$ are shown in Fig. 17 for several values of $\kappa ; \kappa=0$ corresponds to the discontinuous step in the height field.

In Fig. 18 we plot, as a function of $\kappa$, the time required for a leading ${ }^{4}$ rightward traveling bore to form for three different cases of $\alpha$. We determine that a bore has formed when the total energy in the domain begins to decrease (Houghton 1969). If the decrease is too small to be measured, we measure instead the maximum in the slope of the height field and consider a jump in this quantity to signify bore formation. The two measures agree with one another when bores form, but the second measure becomes less precise for very small bores. Note that all bores that were observed to form subsequently decayed.

${ }^{4}$ A secondary bore sometimes forms for $\alpha=.7$ when a leading one does not. 
TABLE 1 . Errors between CLAWPACK and Stoker's analytical solution for the dam-break problem. $c_{s}$ is the bore speed while $\bar{\eta}$ and $\bar{u}$ are the perturbation height and velocity values in the region behind the bore. Values are rounded to five places after the decimal point.

\begin{tabular}{|c|c|c|c|c|c|c|c|c|c|}
\hline \multirow[b]{2}{*}{$\alpha$} & \multicolumn{3}{|c|}{$c_{s}$} & \multicolumn{3}{|c|}{$\bar{\eta}$} & \multicolumn{3}{|c|}{$\bar{u}$} \\
\hline & Analyt. & CLAW & $\%$ error & Analyt. & CLAW & $\%$ error & Analyt. & CLAW & $\%$ error \\
\hline .1 & 1.02540 & 1.02417 & 0.12011 & -.02556 & -.02556 & 0.00990 & 1.00175 & 1.00175 & 0.00033 \\
\hline .3 & 1.08043 & 1.08000 & 0.03971 & -.08191 & -.08190 & 0.00940 & 1.01692 & 1.01692 & 0.00078 \\
\hline .5 & 1.14737 & 1.14667 & 0.06111 & -.15142 & -.15142 & 0.00198 & 1.05338 & 1.05338 & 0.00037 \\
\hline .7 & 1.24354 & 1.24125 & 0.18383 & -.25022 & -.25023 & 0.00446 & 1.13038 & 1.13038 & 0.00112 \\
\hline
\end{tabular}

The three solid curves in Fig. 18 show that bores do form even when the initial conditions are smooth and, as expected, the time required for bore formation increases as the profile becomes smoother. It can also be seen that for $\kappa$ roughly less than 3 the curves tend to overlap, indicating that $\kappa$ is a good measure of bore formation (i.e., no $\alpha$ dependence in this regime). Also from the Fig. 18 one can deduce that leading bores will not form for $\mathcal{L}>(.6,1.25,1.75)$ for $\alpha=(.1, .4, .7)$ respectively.

In the spirit of Houghton (1969) and Williams and Hori (1970) [and most recently Fedorov and Melville (1995) for the case of a Kelvin wave], we have also plotted in Fig. 18 (dotted curves) the time of bore formation in the case when rotation is absent $(\epsilon=0)$; these dotted curves demonstrate that bore formation is delayed when rotation is added. Note that all three curves coincide for all the times considered here, suggesting similarity behavior. After plotting these curves, it is simple to understand why all (dotted and solid) curves overlap for $\kappa$ roughly less than 3: the length scale $\mathcal{L}<1$ in this regime, and hence the scales involved here, are smaller than a deformation radius. Hence rotation has little effect. In general, as $\alpha$ decreases for constant $\kappa$, the solid curves will approaches the dotted curves for this same reason.

\section{Discussion}

In the last review paper that has appeared on the subject of geostrophic adjustment, Blumen (1972) emphasized the following:

In principle, the conservation of potential vorticity provides a means for the determination of steady-state field properties that evolve from a given initial state. Rossby's solution ... provides a case in point. Yet Rossby's solution can never be realized if, for example, the formation of hydrodynamic instability leads to turbulent dissipation of energy before a steady state is established. This possibility is suggested in the studies of Tepper (1955) and Houghton (1969).

In this paper, we have attempted to address the problem of the realization of the "Rossby solution" in the case when the "hyrodynamic instability" is a bore. We have extended the work of Tepper and Houghton to full nonlinearity and have integrated past the time when discontinuous solutions first form. In a nutshell, our results indicate that the Rossby solution is, in fact, very nearly realized because the dissipative effects of bores are either negligible, or rapidly become negligible, due to their decay in a rotating environment. We caution, however, that the energy loss due to bores [quantified in (18)] is more of a mathematical property of the shallowwater equations than a physically realistic quantity. A parameterization of the "turbulent dissipation of energy" at the head of the bore would be more appropriate.

The idea that rotating environments are not conducive to the formation of bores was widely held until Houghton (1969) showed that under certain circumstances, bores can form. We have corroborated Houghton's results by showing that bores do form even from smooth initial conditions. In addition however, our study has shown that these same bores decay because of rotation. We have found that the decay mechanism is not a simple dispersion, which would smooth out the discontinuities, but rather a steady decay of the amplitude while the fields remain discontinuous.

It is a common belief that geostrophic adjustment is a relatively "fast" process, that is, completes on a timescale of order $1 / f$. A careful reading of the literature (Blumen 1967a, 1972; Middleton 1987; Hermann and Owens 1993) reveals, however, that the timescale for adjustment is highly dependent on initial conditions and can vary greatly. In our problem we have found, in addition, that the adjustment time can be highly dependent on what quantity is used to measure it. Pointwise measures are highly sensitive and can lead to misleading conclusions. The often quoted ratio of the change in kinetic to potential energy, which for our problem is predicted to be $1 / 3$ by conservation methods, may be a poor measure as well. Even after some 83 time periods, this ratio still oscillates by as much as $30 \%$ around the value $^{5}$ of $1 / 3$. Measuring the rms fields gives the correct sense that the height field adjusts much faster-typically in a few inertial periods-compared to the velocity fields, which can take substantially longer to adjust.

\footnotetext{
${ }^{5}$ An anonymous referee has suggested that the energy ratio measure might still be useful if one averaged over an inertial period; in this case one could speak of "geostrophic adjustment of the subinertial state" (i.e., the instantaneous state averaged over an inertial period) as opposed to "geostrophic adjustment of the instantaneous state." The adjustment of the former would be much faster than the adjustment of the latter.
} 
Finally, we have carefully examined the effects of nonlinearity and rotation on bores and have found that, in our problem, bores tend to decay faster when the rotation and/or the nonlinearity increases. We have also performed a few initial value problems with Rossby's original initial conditions (i.e., a jet in $v$ and a flat $h$ at $t=0$ ) and have found similar results. Both problems, however, are extremely simplified. The question of whether and how much our results carry over to more realistic (stratified ${ }^{6}$, two-dimensional, etc.) conditions remains open.

Acknowledgments. We are grateful to Drs. William Blumen, Mark Cane, C.K. Chu, Bjorn Enquist, Rupert Ford, Joseph Klemp, Dick Ou, Richard Rotunno, Chi Wang Shu, and William Skamarock for helpful suggestions. The CLAWPACK Riemann solver used in this study was based on source code provided by Drs. Karl Helfrich and Randy Leveque. Linear adjustment computations were performed on the Pittsburg Supercomputing Center's Cray C90. This work is supported by the National Science Foundation.

\section{APPENDIX A}

\section{The Numerical Method}

We integrate Eq. (4) using a Roe approximate Riemann solver with second-order corrections. The specific implementation we use is called CLAWPACK (CONservation LAWs software package, Leveque 1995). Details on this method are given in either the CLAWPACK documentation or Leveque (1992). We briefly describe the method here.

A system of conservation laws in one space dimension

$$
q_{t}+f(q)_{x}=0
$$

may rewritten as

$$
q_{t}+A(q) q_{x}=0
$$

where $q$ is a vector of dependent variables and $A(q)=$ $\partial f / \partial q$ denotes the Jacobian matrix of the flux vector $f$. The key idea is that discretization of the above system leads to the viewpoint that $q(x)$ is locally piecewise constant, thus defining a series of local (solvable) Riemann problems. In other words, discretizing by taking $x \rightarrow x_{i}$ with grid spacing $\Delta x$, we define the $i$ th local Riemann problem to be centered at $x_{i}+\Delta x / 2$ having left and right states given by $q\left(x_{i}\right)$ and $q\left(x_{i+1}\right)$, which we denote by $q_{l}$ and $q_{r}$. Now the solution to Riemann problems typically requires some iteration procedure to solve the nonlinear, algebraic equations that arise [for example, the shallow-water case requires a solution of (9)].

\footnotetext{
${ }^{6}$ As of this date, W. Blumen and R. Williams were finishing work on the stratified problem.
}

It turns out that a simplification may be made. Instead of solving local nonlinear Reimann problems exactly, one replaces $A(q)$ by a new constant coefficient matrix, $\hat{A}$, and thus solve local linearized Riemann problems. What is this new matrix $\hat{A}$ ? It should be clear that it must be some function of the left and right states $q_{l}$ and $q_{r}$. Three conditions were given on $\hat{A}$ (Roe 1981; Leveque 1992), the so-called Roe matrix:

1) $\hat{A}\left(q_{l}, q_{r}\right)\left(q_{r}-q_{l}\right)=f\left(q_{r}\right)-f\left(q_{l}\right)$.

2) $\hat{A}\left(q_{l}, q_{r}\right)$ is diagonalizable with real eigenvalues.

3) $\hat{A}\left(q_{l}, q_{r}\right) \rightarrow A(\bar{q})$ smoothly as $q_{l}, q_{r} \rightarrow \bar{u}$.

Godunov's method, an upwind type scheme based on a decomposition of the new linear problem into characteristic fields, is applied to the new equations. To improve the accuracy of this first-order scheme, a secondorder correction is added to the flux term based on local slopes (the "flux" or "slope" limiter) of the solution.

In the rotating shallow-water equations, a Coriolis source term must be added to the right hand side of (A2), denoted by $\psi(q)$. This additional term is handled using a splitting method (Strang splitting). In this method, one alternates between solving the homogeneous equation $q_{t}+F(q)_{x}=0$ and $q_{t}=\psi(q)$. Finally, because the linear equations cannot capture rarefaction waves, a so-called "entropy fix" must be applied.

As for numerical accuracy, the method we use is second-order in time and second order in space in smooth regions of the flow. Near discontinuities, the spatial accuracy reduces to first order. It is possible to obtain thirdand fourth- order accuracy in time and fourth- and fifthorder accuracy in space (in smooth regions) by using other flux limiting corrections; two common methods are the ENO (essentially nonoscillatory, Harten et al. 1987) and WENO (weighted essentially non-oscillatory, Liu et al. 1994) schemes. We have found the secondorder accurate scheme to be sufficient for the present study.

To test CLAWPACK, we use the exact analytical solution (8) for the dam-break problem. Figure A1 shows selected data points (diamonds and crosses) superimposed on solution (8) for the $\eta$ and $u$ fields in a typical integration, for the case $\alpha=.5$ at time $t=40$. Table 1 gives numerical values for the bore speed $c_{s}$, perturbation height $\bar{\eta}$, and the velocity behind the bore $\bar{u}$ as the parameter $\alpha$ is varied for the numerical resolution used throughout this paper. Errors are typically of the order less than $0.1 \%$; CLAWPACK performs well on this problem, smearing the bore over a constant $\approx 5$ grid spaces throughout the integration.

We also tried a number of flux limiters and noticed only a slight change in the plots of the computed solutions. "Superbee" and "monotonized center" flux limiters seemed to give the sharpest PV fronts but tended to increase numerical dissipation. The opposite effect was found with the "minmod" limiter. 


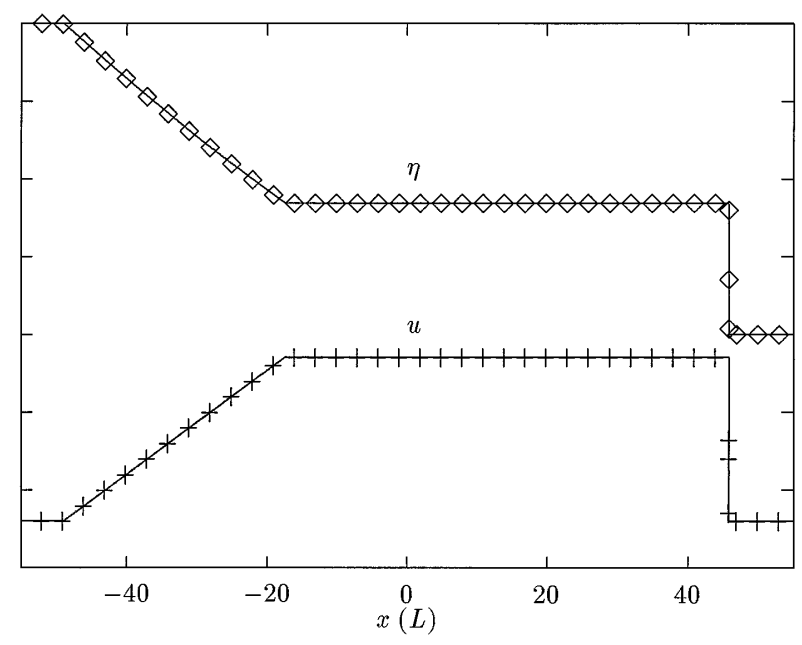

FIG. A1. CLAWPACK test: perturbation height $\eta$ and velocity $u$ for the dam break problem at $t=40(L / \sqrt{g H})$. Selected numerical values (+'s and $\diamond$ 's) are plotted versus the analytical (-) solution for $\alpha=.5$.

APPENDIX B

\section{Asymptotic Solution in the $\epsilon \ll 1$ Limit}

Substitution of (13) into Eq. (4) yields the following sequence of problems.

At $O(1)$ :

$$
\begin{aligned}
\frac{\partial u^{(0)}}{\partial t}+\alpha\left(u^{(0)} \frac{\partial u^{(0)}}{\partial x}\right)+\frac{\partial \eta^{(0)}}{\partial x} & =0 \\
\frac{\partial \boldsymbol{v}^{(0)}}{\partial t}+\alpha\left(u^{(0)} \frac{\partial v^{(0)}}{\partial x}\right) & =0 \\
\frac{\partial \eta^{(0)}}{\partial t}+\frac{\partial u^{(0)}}{\partial x}+\alpha\left(\frac{\partial\left(\eta^{(0)} u^{(0)}\right)}{\partial x}\right) & =0
\end{aligned}
$$

At $O(\epsilon)$ :

$$
\begin{aligned}
\frac{\partial u^{(1)}}{\partial t}+\alpha\left(u^{(0)} \frac{\partial u^{(1)}}{\partial x}+u^{(1)} \frac{\partial u^{(0)}}{\partial x}\right)+\frac{\partial \eta^{(1)}}{\partial x} & =v^{(0)} \\
\frac{\partial v^{(1)}}{\partial t}+\alpha\left(u^{(0)} \frac{\partial v^{(1)}}{\partial x}+u^{(1)} \frac{\partial v^{(0)}}{\partial x}\right) & =-u^{(0)} \\
\frac{\partial \eta^{(1)}}{\partial t}+\frac{\partial u^{(1)}}{\partial x}+\alpha\left(\frac{\partial\left(\eta^{(0)} u^{(1)}\right)}{\partial x}+\frac{\partial\left(\eta^{(1)} u^{(0)}\right)}{\partial x}\right) & =0
\end{aligned}
$$

At $O\left(\epsilon^{2}\right)$ :

$$
\begin{aligned}
& \frac{\partial u^{(2)}}{\partial t}+\alpha\left(u^{(0)} \frac{\partial u^{(2)}}{\partial x}+u^{(2)} \frac{\partial u^{(0)}}{\partial x}\right)+\frac{\partial \eta^{(2)}}{\partial x} \\
& =v^{(1)}-\alpha u^{(1)} \frac{\partial u^{(1)}}{\partial x} \\
& \frac{\partial v^{(2)}}{\partial t}+\alpha\left(u^{(0)} \frac{\partial v^{(2)}}{\partial x}+u^{(2)} \frac{\partial v^{(0)}}{\partial x}\right) \\
& =-u^{(1)}-\alpha u^{(1)} \frac{\partial v^{(1)}}{\partial x} \\
& \frac{\partial \eta^{(2)}}{\partial t}+\frac{\partial u^{(2)}}{\partial x}+\alpha\left(\frac{\partial\left(\eta^{(0)} u^{(2)}\right)}{\partial x}+\frac{\partial\left(\eta^{(2)} u^{(0)}\right)}{\partial x}\right) \\
& =-\alpha \frac{\partial\left(\eta^{(1)} u^{(1)}\right)}{\partial x} .
\end{aligned}
$$

For the $O(1)$ order problem take the initial conditions (5). For $n=1,2,3, \cdots$, the initial conditions are $u^{(n)}$ $=\boldsymbol{v}^{(n)}=\eta^{(n)}=0$ initially and throughout the regions I and IV of the $x-t$ plane of Fig. 6a. The solution of the $O(1)$ problem is given by (8) and is simply the nonrotating dam break solution (Stoker 1958).

At $O(\epsilon)$ the $v$ equation decouples from the other two. Since $v^{(0)}(x, t)=0$ and the boundary conditions are homogeneous, the solutions of (B4) and (B6) are $u^{(1)}(x$, $t)=\eta^{(1)}(x, t)=0$. In fact, at each order, the $y$-momentum equation decouples from $x$-momentum and continuity equation, implying that $\eta^{(2 n+1)}(x, t)=u^{(2 n+1)}(x, t)=0$, for $n=0,1,2, \cdots$.

To complete the $O(\epsilon)$ solution, we solve (B5) by the method of characteristics. The full $O(\epsilon)$ solution is then

$$
\begin{aligned}
& u^{(1)}(x, t)=0 \forall(x, t) \\
& \boldsymbol{v}^{(1)}(x, t)= \begin{cases}0 & \text { region I } \\
\frac{1}{\alpha}\left(-x+\frac{\left(x-2 c_{l} t\right)^{3}}{27 c_{l}^{2} t^{2}}\right) & \text { region II } \\
\frac{1}{\alpha}\left(-x+\frac{(x-\alpha \bar{u} t)\left(\bar{c}-\alpha \bar{u}+2 c_{l}\right)^{3}}{27 \bar{c} c_{l}^{2}}\right) & \text { region IIIa } \\
\left(-\bar{u} t+\bar{u} \frac{(x-\alpha \bar{u} t)}{\left(c_{s}-\alpha \bar{u}\right)}\right) & \text { region IIIb } \\
0 & \text { region IV }\end{cases}
\end{aligned}
$$

$\eta^{(1)}(x, t)=0 \forall(x, t)$,

where regions I, II, IV and $c_{l}, \bar{c}, \bar{u}$, and $c_{s}$ are as described in section $3 \mathrm{~b}$. Region III as described in that section needs to be split into two subregions IIIa and IIIb separated by the new characteristic line $x=\alpha u^{(0)} t=\alpha \bar{u} t$ dictated by Eq. (B5).

The solution at $O\left(\epsilon^{2}\right)$ is complicated. In general, $\left.v^{(2 n}\right)$ $=0, n=0,1,2, \cdots$ by the previously mentioned decoupling. To complete the solution one needs to solve Eqs. (B7) and (B9); this can be done in regions I, III, and IV. However, the solution in region III has boundary 
conditions dependent upon the solution in region II, and Eqs. (B7) and (B9) are not easily solved in region II because the system cannot be diagonalized there. Therefore, we make an approximation and extend the known values of $u^{(2)}$ and $\eta^{(2)}$ from region I to region II so as to provide boundary conditions for the equations in region III. This approximation becomes exact as $\alpha \rightarrow 0$. The good agreement between our asymptotic solution and the full numerical solution (see Fig. 16) provides a validation for this approximation.

Since many lower-order terms vanish, the problem for $u^{(2)}$ and $\eta^{(2)}$ in region III reduces to

$$
\begin{aligned}
& \frac{\partial u^{(2)}}{\partial t}+\alpha\left(u^{(0)} \frac{\partial u^{(2)}}{\partial x}\right)+\frac{\partial \eta^{(2)}}{\partial x}=v^{(1)} \\
& \frac{\partial \eta^{(2)}}{\partial t}+\frac{\partial u^{(2)}}{\partial x}+\alpha\left(\eta^{(0)} \frac{\partial u^{(2)}}{\partial x}+u^{(0)} \frac{\partial \eta^{(2)}}{\partial x}\right)=0,
\end{aligned}
$$

which we diagonalize by defining two new variables $A(x, t)$ and $B(x, t)$, to obtain

$$
\begin{aligned}
& \frac{\partial A}{\partial t}+(\alpha \bar{u}+\bar{c}) \frac{\partial A}{\partial x}=\bar{c} \boldsymbol{v}^{(1)} \\
& \frac{\partial B}{\partial t}+(\alpha \bar{u}-\bar{c}) \frac{\partial B}{\partial x}=\bar{c} \boldsymbol{v}^{(1)},
\end{aligned}
$$

where

$$
\begin{aligned}
& A(x, t) \equiv \bar{c} u^{(2)}(x, t)+\eta^{(2)}(x, t) \\
& B(x, t) \equiv \bar{c} u^{(2)}(x, t)-\eta^{(2)}(x, t) .
\end{aligned}
$$

For well-posed boundary conditions on Eqs. (B13) and (B14), we take $A=0$ only on the line $x=\alpha \bar{u}-\bar{c} t$ and $B=0$ only on the line $x=c_{s} t$. The solutions in region III then become

$$
\begin{aligned}
& A(x, t)= \begin{cases}A^{\mathrm{III}}(x, t) & \text { region IIIa } \\
A^{\mathrm{IIb}}(x, t) & \text { region IIIb }\end{cases} \\
& B(x, t)= \begin{cases}B^{\mathrm{III}}(x, t) & \text { region IIIa } \\
B^{\mathrm{IIl}}(x, t) & \text { region IIIb }\end{cases}
\end{aligned}
$$

with

$$
\begin{aligned}
A^{\mathrm{III}}(x, t)=\frac{\bar{c}}{\alpha}\left[\frac{1}{2}[\right. & \left.-\bar{c}-\alpha \bar{u}+\frac{1}{27 c_{l}^{2}}\left(\bar{c}-\alpha \bar{u}+2 c_{l}\right)^{3}\right] \\
& \left.\times\left(t^{2}-t_{1}^{2}(x, t)\right)\right] \\
+\frac{\bar{c}}{\alpha}[[ & {\left[2 \bar{c}-\frac{2}{27 c_{l}^{2}}\left(\bar{c}-\alpha \bar{u}+2 c_{l}\right)^{3}\right] } \\
& \left.\times\left(t-t_{1}(x, t)\right) t_{1}(x, t)\right]
\end{aligned}
$$

$$
\begin{aligned}
& A^{\mathrm{IIIb}}(x, t)=A^{\mathrm{IIIa}}\left(x, t_{2}(x, t)\right) \\
& +\frac{\bar{c}}{\alpha}\left[\frac{1}{2}\left(\frac{\alpha \bar{u} \bar{c}}{\mathrm{c}_{s}-\alpha \bar{u}}-\alpha \bar{u}\right)\left(t^{2}-t_{2}^{2}(x, t)\right)\right] \\
& -\frac{\bar{c}}{\alpha}\left[2 \bar{c} t_{0}\left[\frac{\alpha \bar{u}}{c_{s}-\alpha \bar{u}}\right]\left(t-t_{2}(x, t)\right)\right] \\
& B^{\mathrm{IIb}}(x, t)=\frac{\bar{c}}{\alpha}\left[\frac{1}{2}\left[-\alpha \bar{u}-\frac{\alpha \bar{u}}{c_{s}-\alpha \bar{u}}\right]\left(t^{2}-t_{3}^{2}(x, t)\right)\right] \\
& +\frac{\bar{c}}{\alpha}\left[\left[\left(\frac{\alpha \bar{u} \bar{c}}{c_{s}-\alpha \bar{u}}\right)\left(\bar{c}-\alpha \bar{u}+c_{s}\right)\right]\right. \\
& \left.\times\left(t-t_{3}(x, t)\right) t_{3}(x, t)\right]
\end{aligned}
$$

$B^{\mathrm{III}}(x, t)=B^{\mathrm{IIl}}\left(x, t_{4}(x, t)\right)$

$$
\begin{aligned}
& +\frac{\bar{c}}{\alpha}\left[\frac{1}{2}\left[\bar{c}-\alpha \bar{u}-\frac{1}{27 c_{l}^{2}}\left(\bar{c}-\alpha \bar{u}+2 c_{l}\right)^{3}\right]\right. \\
& \left.\times\left(t^{2}-t_{4}^{2}(x, t)\right)\right] \\
& +\frac{\bar{c}}{\alpha}\left[\left[\left(\bar{c}-\alpha \bar{u}+c_{s}\right)\right.\right. \\
& \left.\times\left(-1+\frac{1}{27 \bar{c} c_{l}^{2}}\left(c_{s}-\alpha \bar{u}+2 c_{l}\right)^{3}\right)\right] \\
& \left.\times\left(t-t_{4}(x, t)\right) t_{3}(x, t)\right],
\end{aligned}
$$

and with

$$
\begin{aligned}
& t_{1}(x, t)=\frac{1}{2 \bar{c}}(-x+(\alpha \bar{u}+\bar{c}) t) \\
& t_{2}(x, t)=2 t_{0}(x, t) \\
& t_{3}(x, t)=\frac{x-(\alpha \bar{u}+\bar{c}) t}{c_{s}-\alpha \bar{u}+\bar{c}} \\
& t_{4}(x, t)=\frac{1}{\bar{c}}(x-(\alpha \bar{u}+\bar{c}) t) .
\end{aligned}
$$

With the solution for $A(x, t)$ and $B(x, t)$, we can now invert (B17) and (B18) to obtain $u^{(2)}$ and $\eta^{(2)}$ in region III. Writing everything out, the $O\left(\epsilon^{2}\right)$ approximate solution is

$\boldsymbol{v}^{(2)}(x, t)=0 \forall(x, t)$ 


$$
\begin{aligned}
& u^{(2)}(x, t) \\
& = \begin{cases}0 & \text { region I } \\
0 & \text { region II } \\
\frac{1}{2 \bar{c}}\left(A^{\mathrm{III}}(x, t)+B^{\text {III }}(x, t)\right) & \text { region IIIa } \\
\frac{1}{2 \bar{c}}\left(A^{\mathrm{IIb}}(x, t)+B^{\mathrm{IIb}}(x, t)\right) & \text { region IIIb } \\
0 & \text { region IV }\end{cases}
\end{aligned}
$$

$$
\begin{aligned}
& \eta^{(2)}(x, t) \\
& = \begin{cases}0 & \text { region I } \\
0 & \text { region II } \\
\frac{1}{2}\left(A^{\text {III }}(x, t)-B^{\text {IIIa }}(x, t)\right) & \text { region IIIa } \\
\frac{1}{2}\left(A^{\mathrm{IIb}}(x, t)-B^{\mathrm{III}}(x, t)\right) & \text { region IIIb } \\
0 & \text { region IV },\end{cases}
\end{aligned}
$$

where $A^{\text {IIIa }}, A^{\text {III }}, B^{\text {IIIa }}, B^{\text {III }}$ are given by (B21), (B22), (B23), and (B24). Evaluating the above on the line $x=$ $c_{s} t$, one may obtain the rate of amplitude decay of the initial right-going bore; the expression for this is given in Eq. (17).

\section{REFERENCES}

Blumen, W., 1967a: Energy partition in a barotropic atmosphere. Tellus, XIX, 174-182.

— 1967b: On nonlinear geostrophic adjustment. J. Atmos. Sci., 24, 325-332.

, 1972: Geostrophic adjustment. Rev. Geophys. Space Phys., 10, 485-528.

Bolin, B., 1953: The adjustment of a non-balanced velocity field toward geostrophic equilibrium in a stratified fluid. Tellus, 5, 373-385.

Boss, B., and L. Thompson, 1995: Energetics of nonlinear geostrophic adjustment. $J$. Phys. Oceanogr., 25, 1521-1529.

Cahn, A., 1945: An investigation of the free oscillations of a simple current system. J. Meteor., 2, 113-119.

Csanady, G. T., 1971: On the equilibrium shape of the thermocline in a shore zone. J. Phys. Oceanogr., 1, 263-270.

- 1979: The birth and death of a warm core ring. J. Geophys. Res., 84, 777-780.

Fedorov, A., and K. Melville, 1995: Propagation and breaking of nonlinear Kelvin waves. J. Phys. Oceanogr., 25, 2518-2531.

Flierl, G. R., 1979: A simple model for the structure of warm and cold core rings. J. Geophys. Res., 84, 781-785.

Friedrichs, K. O., 1948: Formation and decay of shock waves. Commun. Pure Appl. Math., 1 (3), 211-245.

Gill, A. E., 1976: Adjustment under gravity in a rotating channel. $J$. Fluid Mech., 103, 275-295.

- 1982: Atmosphere-Ocean Dynamics. Academic Press, 662 pp.

Glendening, J. W., 1993: Nonlinear displacement of the geostrophic velocity jet created by mass imbalance. J. Atmos. Sci., 50, 16171628.

Harten, A., B. Enquist, S. Osher, and S. K. Chakravarthy, 1987: Uniformly high order accurate essentially non-oscillatory shemes, III. J. Comput. Phys., 71, 231-303.

Hermann, A. J., and W. B. Owens, 1993: Energetics of gravitational adjustment in mesoscale chimneys. J. Phys. Oceanogr., 23, 346371.

Houghton, D. D., 1969: Effect of rotation on the formation of hydraulic jumps. J. Geophys. Res., 74, 1351-1360.

Hsueh, Y., and B. Cushman-Roisin, 1983: On the formation of surface to bottom fronts over steep topography. J. Geophys. Res., 88, $743-750$.

Killworth, P. D., 1992: The time-dependent collapse of a rotating fluid cylinder. J. Phys. Oceanogr., 22, 390-397.

Klemp, J., R. Rotunno, and W. C. Skamarock, 1997: On the propagation of internal bores. J. Fluid Mech., 331, 81-106.

Lax, A., 1948: Decaying shocks. Commun. Pure Appl. Math., 1 (3), 247-257.

Lax, P. D., and B. Wendroff, 1960: Systems of conservation laws. Commun. Pure Appl. Math., 13, 217-237.

Leveque, R. J., 1992: Numerical Methods for Conservation Laws. Birkhauser, $214 \mathrm{pp}$.

, 1995: CLAWPACK CONservation LAWs software package.

Liu, X. D., S. Osher, and T. Chan, 1994: Weighted essentially nonoscillatory schemes. J. Comput. Phys., 115, 200-212.

McWilliams, J. C., 1988: Vortex generation through balanced adjustment. J. Phys. Oceanogr., 18, 1178-1192.

Middleton, J. F., 1987: Energetics of linear geostrophic adjustment. J. Phys. Oceanogr., 17, 735-740.

Mihaljan, J. M., 1963: The exact solution of the Rossby adjustment problem. Tellus, 15, 150-154.

Obukhov, A. M., 1949: On the question of the geostrophic wind. Izv. Akad. Nauk. SSSR Ser. Geograf.-Geofiz. 13 (4), 281-306.

Orlanski, I., 1976: A simple boundary condition for unbounded hyperbolic flows. J. Comput. Phys., 21, 251-269.

Ou, H. W., 1983: Some two-layer models of the shelf slope front: Geostrophic adjustment and it's maintenance. J. Phys. Oceanogr., 13, 1798-1808.

— 1984: Geostrophic adjustment: A mechanism for frontogenesis. J. Phys. Oceanogr., 14, 994-1000.

Pratt, L. J., 1983: On inertial flow over topography. Part 1. Semigeostrophic adjustment to an obstacle. J. Fluid Mech., 131, 195218.

Riabouchinsky, D., 1932: Sur l'analogie hydraulique des mouvements d'un fluide compressible. Comptes Rendus, 195, p. 998.

Riemann, B., 1858: Uber die Fortpflanzung ebener Luftwellen von endlicher Schwingungsweite. Göttingen Abhandlungen, Vol. viii, p. 43.

Roe, P. L., 1981: Approximate Riemann solvers, parameter vectors, and difference schemes. J. Comput. Phys., 43, 357-372.

Rossby, C. G., 1938: On the mutual adjustment of pressure and velocity distributions in certain simple current systems II. J. Mar. Res., 1, 239-263.

Schubert, W. H., J. J. Hack, P. L. Silva Dias, and S. R. Fulton, 1980: Geostrophic adjustment in an axisymmetric vortex. J. Atmos. Sci., 37, 1464-1484.

Skamarock, W. C., J. B. Klemp, and R. Rotunno, 1996: The diffraction of Kelvin waves and bores at coastal bends. J. Atmos. Sci., 53, 1327-1337.

Stoker, J. J., 1958: Water Waves. John Wiley, 567 pp.

Stommel, H., and G. Veronis, 1980: Barotropic response to cooling. J. Geophys. Res., 85C, 6661-6666.

Tepper, M., 1955: On the generation of pressure jump-lines by the impulsive addition of momentum to simple current systems. $J$. Meteor., 12, 287-297.

van Heijst, G. J. F., 1985: A geostrophic adjustment model of a tidal mixing front. J. Phys. Oceanogr., 15, 1182-1190.

Washington, M. W., 1964: A note on the adjustment towards geostrophic equilibrium in a simple fluid system. Tellus, 4, 530533.

Whitham, G. B., 1974: Linear and Nonlinear Waves. John Wiley, $636 \mathrm{pp}$.

Williams, R. T., and A. M. Hori, 1970: Formation of hydraulic jumps in a rotating system. J. Geophys. Res., 75, 2813-2821. 